\title{
THE LIFTING PROBLEM FOR AFFINE STRUCTURES IN NILPOTENT LIE GROUPS
}

\author{
NGUIFFO B. BOYOM
}

\begin{abstract}
Affine manifolds occur in several situations in pure and applied mathematics, (e.g. leaves of Lagrangian foliations, completely integrable Hamiltonian systems, linear representations of virtually polycyclic groups, geometric quantization and so on). This work is devoted to left invariant affinely flat structures in Lie groups. We are mainly concerned with the following situation. Let $G$ and $G_{0}$ be nilpotent Lie groups of dimension $n+1$ and $n$, respectively and let $h: G \rightarrow G_{0}$ be a continuous homomorphism from $G$ onto $G_{0}$. Given a left invariant affinely flat structure $\left(G_{0}, \nabla_{0}\right)$ the lifting problem is to discover whether $G$ has a left invariant affinely flat structure $(G, \nabla)$ such that $h$ becomes an affine morphism. In the present work we answer positively when $\left(G_{0}, \nabla_{0}\right)$ is "normal". Therefore the existence problem for a left invariant complete affinely flat structure in nilpotent Lie groups is solved by applying the following subsequent results. Let $\mathfrak{A} f\left(G_{0}\right)$ be the set of left invariant affinely flat structures in the nilpotent Lie group $G_{0},\left(1^{\circ}\right) \mathfrak{A} f\left(G_{0}\right) \neq \varnothing$ implies the existence of normal structure $\left(G_{0}, \nabla_{0}\right) \in \mathfrak{A} f\left(G_{0}\right) ;\left(2^{\circ}\right) h: G \rightarrow G_{0}$ being as above every normal structure $\left(G_{0}, \nabla_{0}\right)$ has a normal lifted in $\mathfrak{A} f(G)$.
\end{abstract}

\section{INTRODUCTION}

Let $G$ be a real, connected, nilpotent Lie group with the Lie algebra $\mathfrak{g}$. Let $\nabla$ be a left invariant linear connection in the Lie group $G$. We assume that the linear connection $\nabla$ is locally flat and complete; then the couple $(G, \nabla)$ is called an affine structure in the Lie group $G$. Let us denote by $\mathfrak{A} f(G)$ the set of all affine structures in the Lie group $G$. If $\mathfrak{A} f(G)$ is nonempty then every $(G, \nabla) \in \mathfrak{A} f(G)$ will give rise to the linear representation of $\mathfrak{g}$ in itself, $\rho: \mathfrak{g} \rightarrow \mathfrak{g} l(\mathfrak{g})$ which is given by the formula

$$
\rho(X) \cdot Y=\nabla_{X} Y
$$

Since $\nabla$ is locally flat we get the following identities, $\rho_{\nabla}(X) \cdot Y-\rho_{\nabla}(Y)$. $X-[X, Y]=0, \rho_{\nabla}(X) \rho_{\nabla}(Y) \cdot Z-\rho_{\nabla}(Y) \rho_{\nabla}(X) \cdot Z-\rho_{\nabla}([X, Y]) \cdot Z=0$, for

Received by the editors March 25, 1985 and, in revised form, January 4, 1988. Parts of the contents of this paper have been presented to the 1984 AMS-IMS-SIAM Joint Summer Research Conference in the Mathematical Sciences at Brunswick (Massachusetts) sponsored by the N.S.F.

1980 Mathematics Subject Classification (1985 Revision). Primary 53C05, 22E50; Secondary 22E27.

Key words and phrases. Affine structure, Koszul-Vinberg structure, truncated Lie algebra, truncated affine structure. 
$(X, Y, Z) \in \mathfrak{g} \times \mathfrak{g} \times \mathfrak{g}$. The dual representation $\rho_{\nabla}^{*}: \mathfrak{g} \rightarrow \mathfrak{g} l\left(\mathfrak{g}^{*}\right)$ of $\mathfrak{g}$ in its dual space $\mathfrak{g}^{*}$ is obtained by setting

$$
\left(\rho_{\nabla}^{*}(X) \theta\right)(Y)=-\theta\left(\rho_{\nabla}(X) \cdot Y\right) .
$$

We consider the cochain complex $C_{\rho_{\nabla}}\left(\mathfrak{g}, \mathfrak{g}^{*}\right)$ associated to the $\mathfrak{g}$-module $\mathfrak{g}^{*}$. Let $C(G, \mathbf{R})$ be the scalar cochain complex of the Lie group $G[5,11]$. Since the vector space $C(G, \mathbf{R})$ is the exterior algebra $\wedge \mathfrak{g}^{*}$, we get the antisymmetrization operator:

$$
\partial: C_{\rho_{\nabla}}\left(\mathfrak{g}, \mathfrak{g}^{*}\right) \rightarrow \bigwedge \mathfrak{g}^{*},
$$

which maps $\Lambda^{p} \mathfrak{g}^{*} \otimes \mathfrak{g}^{*}$ onto $\Lambda^{p+1} \mathfrak{g}^{*}$. The map $\partial$ is coherent with the cochain complex structures, so that we get the linear map

$$
\partial^{p}: H_{\rho_{\nabla}}^{p}\left(\mathfrak{g}, \mathfrak{g}^{*}\right) \rightarrow H^{p+1}(G, \mathbf{R}) .
$$

THE LIFTING PROBLEM. Let us suppose that $\mathfrak{A} f(G)$ is nonempty. Can one find $(G, \nabla) \in \mathfrak{A} f(G)$ such that $\partial^{1}\left(H_{\rho_{\nabla}}^{1}\left(\mathfrak{g}, \mathfrak{g}^{*}\right)\right)=H^{2}(G, \mathbf{R})$ ?

At the 1984 AMS-IMS-SIAM Summer Research Conferences in the Mathematical Sciences, I sketched a proof of the following result:

Theorem. The vector space $H^{2}(G, \mathbf{R})$ is spanned by $\partial^{1}\left(H_{\rho_{\nabla}}^{1}\left(\mathfrak{g}, \mathfrak{g}^{*}\right)\right)$ when $(G$, $\nabla)$ runs over $\mathfrak{A} f(G)$.

In the present work I will prove the following result.

The lifting problem. Let $G$ be a connected nilpotent Lie group with the Lie algebra $\mathfrak{g}$. Suppose that $\mathfrak{A} f(G)$ is nonempty. Then there is $(G, \nabla) \in \mathfrak{A} F(G)$ such that $\partial^{1}\left(H_{\rho_{\nabla}}^{1}\left(\mathfrak{g}, \mathfrak{g}^{*}\right)\right)=H^{2}(G, \mathbf{R})$.

Remark. The Lifting Theorem gives an alternative proof of the existence theorem for affine structures in nilpotent Lie groups, [9]. Indeed, every nilpotent Lie group is a central extension of a nilpotent Lie group; the Lifting Theorem shows that one obtains (by induction with respect to $\operatorname{dim} G$ ) the existence theorem for affine structures in every nilpotent Lie group, (see Proposition 1.1).

\section{BACKGROUND MATERIAL}

1.1. Koszul-Vinberg-structure. Let $\mathfrak{g}$ be a finite dimensional Lie algebra. Let $\rho$ be a linear representation of $\mathfrak{g}$ in itself with the following properties: for every $(X, Y, Z)$ in $\mathfrak{g} \times \mathfrak{g} \times \mathfrak{g}$ we have

$$
\begin{gathered}
\rho(X) Y-\rho(Y) X-[X, Y]=0 \\
\rho(X) \rho(Y) Z-\rho(Y) \rho(X) Z-\rho[X, Y] Z=0 .
\end{gathered}
$$

Definition 1.1. A Koszul-Vinberg structure in the Lie algebra $\mathfrak{g}$ is a linear representation $\rho: \mathfrak{g} \rightarrow \mathfrak{g} l(\mathfrak{g})$ with the properties $\left(\mathrm{K}_{1}\right)$ and $\left(\mathrm{K}_{2}\right)$.

We denote by $\operatorname{KV}(\mathfrak{g})$ the set of Koszul-Vinberg structures in $\mathfrak{g}$. A KoszulVinberg structure $\rho \in \mathrm{KV}(\mathfrak{g})$ is complete if for every fixed $Y_{0} \in \mathfrak{g}$ the linear 
map $X \rightarrow \psi_{Y_{0}}(X)=\rho(X) Y_{0}+X$ is a regular linear map of $\mathfrak{g}$ in itself. It is known that only solvable Lie algebras can admit complete Koszul-Vinberg structures [5].

One sees that every affine structure $(G, \nabla)$ gives rise to unique complete Koszul-Vinberg structure; conversely, let $\mathfrak{g}$ be a solvable Lie algebra with a complete Koszul-Vinberg structure $\rho$; let $G$ be the connected and simply connected Lie group with the Lie algebra $\mathfrak{g}$. Then there is a unique affine representation of $G$ in the vector space $\mathfrak{g}, s \rightarrow(q(s), f(s)) \in \mathfrak{g} \times \mathrm{GL}(\mathfrak{g})$ such that

$$
d(q, f)_{e}=\left(\mathbf{1}_{\mathfrak{g}}, \rho\right) .
$$

In other words, let $X \in \mathfrak{g}$ and let $\exp t X$ be the one parameter subgroup which is generated by $X$, then for every $Y \in \mathfrak{g}$ we have

$$
\lim _{t \rightarrow 0} \frac{f(\exp t X) \cdot Y-Y}{t}=\rho(X) Y, \quad \lim _{t \rightarrow 0} \frac{q(\exp t X)}{t}=X .
$$

1.2. The Chevalley-Koszul cohomology spaces. In the following we deal with cohomology space of the Lie group $G$ with coefficients in $G$-modules.

Let $\rho$ be a linear representation of the Lie algebra $\mathfrak{g}$ in the vector space $V$. We denote $C_{\rho}(\mathfrak{g}, V)$ the cochain complex which is defined as follows (cf. [3, 11]):

$$
C_{\rho}(\mathfrak{g}, V)=\bigoplus_{p} \operatorname{Hom}\left(\bigwedge^{p} \mathfrak{g}, V\right) .
$$

We set $C_{\rho}^{p}(\mathfrak{g}, V)=\operatorname{Hom}\left(\bigwedge^{p} \mathfrak{g}, V^{p}\right)$. The coboundary operator

$$
\delta: C_{\rho}^{p}(\mathfrak{g}, V) \rightarrow C_{\rho}^{p+1}(\mathfrak{g}, V)
$$

is defined by setting

$$
\begin{aligned}
\delta \varphi\left(X_{0}, \ldots, X_{p}\right)= & \sum_{i}(-1)^{i} \rho\left(X_{i}\right) \varphi\left(X_{0}, \ldots, \widehat{X}_{i}, \ldots, X_{p}\right) \\
& +\sum_{k<j}(-1)^{i+j} \varphi\left(\left[X_{i}, X_{j}\right], \ldots, \widehat{X}_{i}, \ldots, \widehat{X}_{j}, \ldots, X_{p}\right) .
\end{aligned}
$$

One easily verifies that $\delta^{2}=0$. Let

$$
Z_{\rho}^{p}(\mathfrak{g}, V)=\operatorname{Ker}\left(\delta: C_{\rho}^{p}(\mathfrak{g}, V) \rightarrow C_{\rho}^{p+1}(\mathfrak{g}, V)\right)
$$

and

$$
B_{\rho}^{p}(\mathfrak{g}, V)=\delta C_{\rho}^{p-1}(\mathfrak{g}, V) .
$$

The $p$ th $V$-valued cohomology space of $\mathfrak{g}$ is $H_{\rho}^{p}(\mathfrak{g}, V)=Z_{\rho}^{p}(\mathfrak{g}, V) / B_{\rho}^{p}(\mathfrak{g}, V)$.

Let us consider the particular case where $V$ is the trivial $\mathfrak{g}$-module $\mathbf{R}$. Then $C_{0}(\mathfrak{g}, \mathbf{R})$ can be identified with the exterior algebra $\wedge^{*} \mathfrak{g}^{*}$ of the dual space of $\mathfrak{g}$. In that case the cohomology space $H_{0}^{p}(\mathfrak{g}, R)$ is denoted $H^{p}(\mathfrak{g}, \mathbf{R})$.

Let $G$ be a connected Lie group with the Lie algebra $\mathfrak{g}$. Then the cohomology space $H_{0}^{p}(\mathfrak{g}, \mathbf{R})$ is called the $p$ th scalar cohomology space of the Lie group $G$ and we set $H^{p}(G, \mathbf{R})=H^{p}(\mathfrak{g}, \mathbf{R})$. 
1.3. Let us consider a connected Lie group $G$ with the Lie algebra $\mathfrak{g}$. Let $(G, \nabla) \in \mathfrak{A} f(G)$. We define the associated Koszul-Vinberg structure in $\mathfrak{g}$ by setting

$$
\rho_{\nabla}(X) Y=\nabla_{X} Y
$$

for every $(X, Y) \in \mathfrak{g} \times \mathfrak{g}$. The dual representation $\rho_{\nabla}^{*}: \mathfrak{g} \rightarrow \mathfrak{g} l\left(\mathfrak{g}^{*}\right)$ is defined by putting

$$
\left(\rho_{\nabla}^{*}(X) \theta\right)(Y)=-\theta\left(\rho_{\nabla}(X) Y\right)
$$

for every $(\theta, X, Y) \in \mathfrak{g}^{*} \times \mathfrak{g} \times \mathfrak{g}$.

Let us consider the antisymmetrization operator

$$
\partial: \operatorname{Hom}\left(\bigwedge^{p} \mathfrak{g}, \mathfrak{g}^{*}\right) \rightarrow \bigwedge^{p+1} \mathfrak{g}^{*} ;
$$

it induces a cochain morphism $C_{\rho_{\nabla}}\left(\mathfrak{g}, \mathfrak{g}^{*}\right) \rightarrow C(G, R)=C_{0}(\mathfrak{g}, R)$, so that we get a linear map

$$
\partial^{1}: H_{\rho_{\nabla}}^{1}\left(\mathfrak{g}, \mathfrak{g}^{*}\right) \rightarrow H^{2}(G, \mathbf{R})
$$

1.4. Geometrical meaning of the lifting problem. Let $\widetilde{G}$ be a connected nilpotent Lie group with the Lie algebra $\mathfrak{g}$. Let $H$ be a 1-dimensional connected normal subgroup in $\widetilde{G}$. It then follows that $H$ is a central subgroup. Let us assume that $H$ is closed submanifold of $\widetilde{G}$, then the factor group $G=\widetilde{G} / H$ has a Lie group structure such that $1 \rightarrow H \rightarrow \widetilde{G} \rightarrow G \rightarrow \mathbf{1}$ is an exact sequence of connected Lie groups.

If $\mathfrak{g}$ is the Lie algebra of the Lie group $G$, the bracket operation in $\tilde{\mathfrak{g}}$ is obtained by a given 2-dimensional cocycle $\omega \in Z^{2}(\mathfrak{g}, \mathbf{R})$, so that by identifying $\tilde{\mathfrak{g}}$ with the direct product $\mathbf{R} \times \mathfrak{g}$, we have

$$
[(x, X),(y, Y)]=(\omega(X, Y),[X, Y])
$$

for every $((x, X),(y, Y)) \in \tilde{\mathfrak{g}} \times \tilde{\mathfrak{g}}$. Let $(G, \nabla)$ be a complete affine structure in $G$ and let $[\omega] \in H^{2}(G, \mathbf{R})$ be the 2nd cohomology class of $\omega$.

Definition 1.2. One says that the linear connection $\nabla$ has the $[\omega]$-lifting property if $[\omega]$ lies in $\partial^{1}\left(H_{\rho_{\nabla}}^{1}\left(\mathfrak{g}, \mathfrak{g}^{*}\right)\right)$.

If $\nabla$ has the $[\omega]$-lifting property, we can find $\varphi \in Z_{\rho_{\nabla}}^{1}\left(\mathfrak{g}, \mathfrak{g}^{*}\right)$ such that $\partial \varphi=\omega$. In other words, $\varphi$ being a linear map of $\mathfrak{g}$ in $\mathfrak{g}^{*}$, let us set

$$
\varphi(X) \cdot Y=\varphi(X, Y)
$$

the conditions $\delta \varphi=0$ and $\partial \varphi=\omega$ mean that for every $(X, Y, Z)$ in $\mathfrak{g} \times \mathfrak{g} \times \mathfrak{g}$ one has

$$
\begin{gathered}
\varphi(X, Y)-\varphi(Y, X)=\omega(X, Y), \\
\varphi\left(X, \rho_{\nabla}(Y) Z\right)-\varphi\left(Y, \rho_{\nabla}(X) Z\right)-\varphi([X, Y], Z)=0 .
\end{gathered}
$$

Therefore we define a linear map $\tilde{\rho}_{\nabla}: \tilde{\mathfrak{g}} \rightarrow \mathfrak{g} l(\tilde{\mathfrak{g}})$ by setting $\tilde{\rho}_{\nabla}(x, X) \cdot(y, Y)=$ $\left(\varphi(X, Y), \rho_{\nabla}(X) \cdot Y\right)$.

Proposition 1.1. The linear map $\tilde{\rho}_{\nabla}$ defines a complete Koszul-Vinberg structure in the Lie algebra $\tilde{\mathfrak{g}}$. 
Proof. Let $(x, X)$ and $(y, Y)$ be elements in $\mathfrak{g}$. We compute

(i)

$$
\begin{aligned}
\tilde{\rho}_{\nabla}(x, X) \cdot(y \cdot Y)-\tilde{\rho}_{\nabla}(y, Y)(x, X)-[(x, X),(y, Y)] \\
\quad=\left(\varphi(X, Y), \rho_{\nabla}(X) Y\right)-(\varphi(Y, X), \rho(Y) X)-(\omega(X, Y),[X, Y]) \\
\quad=(\varphi(X, Y)-\varphi(Y, X)-\omega(X, Y), \rho(X) Y-\rho(Y) X-[X, Y])=(0,0) .
\end{aligned}
$$

(ii)

$$
\begin{aligned}
\tilde{\rho}_{\nabla}(x, & X) \tilde{\rho}_{\nabla}(y, Y) \cdot(z, Z)-\tilde{\rho}_{\nabla}(y, Y) \tilde{\rho}_{\nabla}(x, X)(z, Z) \\
& -\tilde{\rho}_{\nabla}(\omega(X, Y),[X, Y])(z, Z) \\
= & \tilde{\rho}_{\nabla}(x, X)\left(\varphi(Y, Z), \rho_{\nabla}(Y) Z\right)-\tilde{\rho}_{\nabla}(y, Y)(\varphi(X, Z), \rho(X) Z) \\
& \left.-\left(\varphi([X, Y], Z), \rho_{\nabla}([X, Y]) Z\right) \quad-\quad-\rho_{\nabla}(Y) \rho_{\nabla}(X) Z-\rho_{\nabla}([X, Y]) Z\right) \\
= & \left(\varphi\left(X, \rho_{\nabla}(Y) Z\right)-\varphi\left(Y, \rho_{\nabla}(X) Z\right)-\varphi([X, Y], Z), \rho_{\nabla}(X) \rho_{\nabla}(Y) Z\right. \\
= & (0,0) .
\end{aligned}
$$

(iii) It remains to prove that $\tilde{\rho}_{\nabla}$ is complete. Let $\left(y_{0}, Y_{0}\right)$ be a fixed element in $\tilde{\mathfrak{g}}$ and let $(x, X)$ be an element of $\tilde{\mathfrak{g}}$ such that $\tilde{\rho_{\nabla}}(x, X)\left(y_{0}, Y_{0}\right)+(x, X)=$ $(0,0)$. Then $(x, X)$ is solution of the following system:

$$
\varphi\left(X, Y_{0}\right)+x=0, \quad \rho_{\nabla}(X) Y_{0}+X=0 .
$$

Since $(G, \nabla)$ is a complete affine structure in $G$, the Koszul-Vinberg structure $\rho_{\nabla}$ is complete, so that $\rho_{\nabla}(X) Y_{0}+X=0$ implies $X=0$. It follows that $\tilde{\rho}_{\nabla}$ is a complete Koszul-Vinberg structure in $\tilde{\mathfrak{g}}$.

Now by virtue of Proposition 1.1, we define the affine structure $(\widetilde{G}, \tilde{\nabla})$ in $\widetilde{G}$ by setting $\tilde{\nabla}_{(x, X)}(y, Y)=\tilde{\rho}_{\nabla}(x, X)(y, Y) .(\widetilde{G}, \widetilde{\nabla})$ induces the trivial affine structure $(H, 0)$ in the subgroup $H \subset \widetilde{G}$, so that the exact sequence $1 \rightarrow$ $H \rightarrow \widetilde{G} \rightarrow G \rightarrow 1$ is an exact sequence of affine structures. Therefore, if some $(G, \nabla)$ is such that $\partial^{1}\left(H_{\rho_{\nabla}}^{1}\left(\mathfrak{g}, \mathfrak{g}^{*}\right)\right)=H^{2}(G, \mathbf{R})$, then every central extension of the Lie group $G, \mathbf{1} \rightarrow H \rightarrow \widetilde{G} \rightarrow G \rightarrow \mathbf{1}$, has an affine structure $(\widetilde{G}, \tilde{\nabla})$ which is projected to $(G, \nabla)$. This is the geometrical interpretation of the lifting problem.

1.5. Lower central series of nilpotent Lie group and flag of ideals. Let $G$ be a connected nilpotent Lie group with the Lie algebra $\mathfrak{g}$. We consider the lower central series of the Lie algebra $\mathfrak{g}: C_{0}(\mathfrak{g})=\mathfrak{g}, \ldots, C_{p+1}(\mathfrak{g})=\left[\mathfrak{g}, C_{p}(\mathfrak{g})\right], \ldots$ It is clear that every $C_{p}(\mathfrak{g})$ is an ideal of the Lie algebra $\mathfrak{g}$; moreover, we have the inclusion relation $C_{p+1}(\mathfrak{g}) \subset C_{p}(\mathfrak{g})$. We are concerned with flags $F(\mathfrak{g})=g \supset \cdots \supset \mathfrak{g}_{p} \supset \cdots \supset(0)$ which are finer than the lower central filtration $\mathfrak{g} \supset C_{1}(\mathfrak{g}) \supset \cdots \supset C_{p}(\mathfrak{g}) \supset \cdots \supset(0)$. If $F(\mathfrak{g})=\mathfrak{g} \supset \cdots \supset \mathfrak{g}_{p} \supset \cdots$ is such a flag, then we must have the following:

$$
p=\operatorname{dim} \mathfrak{g}_{p} \leq \operatorname{dim} C_{q}(\mathfrak{g}) \Rightarrow \mathfrak{g}_{p} \subset C_{q}(\mathfrak{g})
$$


and

$$
\operatorname{dim} C_{q}(\mathfrak{g}) \leq p \Rightarrow C_{q}(\mathfrak{g}) \supset \mathfrak{g}_{p}
$$

Lemma 1.1. Every nilpotent Lie algebra has a flag of ideals $F(\mathfrak{g})$ which is finer than the lower central series $\mathfrak{g} \supset C_{1}(\mathfrak{g}) \supset \cdots \supset C_{p}(\mathfrak{g}) \supset C_{p+1}(\mathfrak{g}) \supset \cdots$.

Proof. We will carry out the proof by induction on $\operatorname{dim} \mathfrak{g}$. We observe that the lemma holds if $\mathfrak{g}$ is commutative. Thus let us suppose that the lemma holds for $\operatorname{dim} \mathfrak{g}<n+1$. Let $\mathfrak{g}$ be an $(n+1)$-dimensional nilpotent Lie algebra with $C_{1}(\mathfrak{g}) \neq(0)$.

Let $p_{0}$ be the positive integer such that $C_{p_{0}}(\mathfrak{g}) \neq(0)$ and $C_{p_{0}+1}(\mathfrak{g})=(0)$. Then $C_{p_{0}}(\mathfrak{g})$ is contained in the center of $\mathfrak{g}$. Let $\Delta$ be a 1-dimensional ideal contained in $C_{p_{0}}(\mathfrak{g})$. We take the quotient Lie algebra $\overline{\mathfrak{g}}=\mathfrak{g} / \Delta$. Let $\pi: \mathfrak{g} \rightarrow \overline{\mathfrak{g}}$ be the canonical homomorphism, so that $\pi\left(C_{p}(\mathfrak{g})\right)=C_{p}(\overline{\mathfrak{g}})$. Since $\operatorname{dim} \overline{\mathfrak{g}}=n$, there is a flag of ideals $F(\overline{\mathfrak{g}})=\overline{\mathfrak{g}} \supset \cdots \supset \overline{\mathfrak{g}}_{p_{0}} \supset \cdots$ which is finer than the central series $\overline{\mathfrak{g}} \supset C_{1}(\overline{\mathfrak{g}}) \supset \cdots$. Let us set $\mathfrak{g}_{p+1}=\pi^{-1}\left(\overline{\mathfrak{g}}_{p}\right)$. It is obvious that every $\mathfrak{g}_{p+1}=\pi^{-1}\left(\overline{\mathfrak{g}}_{0}\right)$ is a $(p+1)$-dimensional ideal in $\mathfrak{g}$, so that $F(\mathfrak{g})=\pi^{-1}(F(\overline{\mathfrak{g}}))$ is a flag of ideals. The condition $\pi\left(C_{p}(\mathfrak{g})\right)=C_{p}(\overline{\mathfrak{g}})$ shows that $F(\mathfrak{g})$ is finer that the lower central series $g \supset C_{1}(\mathfrak{g}) \supset \cdots$. Q.E.D.

\section{TRUNCATEd LiE ALgEBRAS AND TRUNCATED AFFINE STRUCTURES}

Let $\mathfrak{g}$ be an $n$-dimensional nilpotent Lie algebra with the fixed flag of ideals $F(\mathfrak{g})=\mathfrak{g} \supset \cdots \supset \mathfrak{g}_{k+1} \supset \mathfrak{g}_{k} \supset \cdots$. Let us fix the following notations:

(i) $E_{F}^{\circ}(\mathfrak{g})$ is the set of nilpotent linear endomorphisms of the vector space $\mathfrak{g}$ which preserve the filtration $F(\mathfrak{g})$;

(ii) $\mathscr{D}_{F}^{\circ}(\mathfrak{g})$ is the subset of $E_{F}^{\circ}(\mathfrak{g})$ defined by the condition that $D \in \mathscr{D}_{F}^{\circ}(\mathfrak{g})$ if and only if $D$ is derivation of the lie algebra $\mathfrak{g}$. That is to say

$$
D[X, Y]=[D X, Y]+[X, D Y]
$$

for every $(X, Y) \in \mathfrak{g} \times \mathfrak{g}$.

Let us consider a pair $(J, I)$ where $I$ is a totally ordered finite set and $J$ is a subset of $I$ with the following property: let $(\gamma, \beta) \in J \times I$ then $\beta \in J$ whenever $\beta \leq \gamma$. We deal with data

$$
K_{J, I}^{F}(\mathfrak{g})=\left\{D_{\alpha}, \xi_{\alpha \beta}, \varepsilon_{\alpha \beta}^{\gamma} /(\alpha, \beta, \gamma) \in I^{3}\right\}
$$

where for every triple $(\alpha, \beta, \gamma)$ one has $\left(D_{\alpha}, \xi_{\alpha \beta}, \varepsilon_{\alpha \beta}^{\gamma}\right) \in \mathscr{D}_{F}^{\circ}(\mathfrak{g}) \times \mathfrak{g} \times \mathbf{R}$; moreover $\left(\xi_{\alpha \beta}, \varepsilon_{\alpha \beta}^{\gamma}\right)$ satisfies the following conditions:

$$
\begin{gathered}
\xi_{\alpha \beta}+\xi_{\beta \alpha}=0, \quad \varepsilon_{\alpha \beta}^{\gamma}+\varepsilon_{\beta \alpha}^{\gamma}=0, \\
\varepsilon_{\alpha \beta}^{\gamma}=0 \quad \text { if } \gamma \geq \min (\alpha, \beta) .
\end{gathered}
$$

We write \#I for the cardinal of the set $I$. 
Definition 2.1. The couple $\left(\mathfrak{g}, K_{J, I}^{F}(\mathfrak{g})\right)$ is called an $(n+\# I)$-dimensional truncated Lie algebra if the following conditions hold:

$\left(c_{\alpha \beta}\right):\left[D_{\alpha}, D_{\beta}\right]=a d \xi_{\alpha \beta}+\sum_{\gamma} \varepsilon_{\alpha \beta}^{\gamma} D_{\gamma}$,

$\left(c_{\alpha \beta \gamma}\right): D_{\alpha} \xi_{\beta \gamma}-D_{\beta} \xi_{\alpha \gamma}+D_{\gamma} \xi_{\alpha \beta}-\sum_{t}\left(\varepsilon_{\beta \gamma}^{t} \xi_{t \alpha}-\varepsilon_{\alpha \gamma}^{t} \xi_{t \beta}+\varepsilon_{\alpha \beta}^{t} \xi_{t \gamma}\right)=0$,

$\left(c_{J, \gamma_{0}}\right)_{\alpha \beta \gamma}$ : for every fixed $\gamma_{0} \in J$, we have

$$
\sum_{t}\left(\varepsilon_{\beta \gamma}^{t} \varepsilon_{t \alpha}^{\gamma_{0}}-\varepsilon_{\alpha \gamma}^{t} \varepsilon_{t \beta}^{\gamma_{0}}+\varepsilon_{\alpha \beta}^{t} \varepsilon_{t \gamma}^{\gamma_{0}}\right)=0
$$

Examples of truncated Lie algebras. Let $\mathfrak{g}_{k}$ be the $k$-dimensional ideal of the flag $F(\mathfrak{g})$. We choose a basis $\left(e_{1}, \ldots, e_{k}, \ldots, e_{n}\right)$ such that every ideal $\mathfrak{g}_{k}$ is spanned by $\left(e_{1}, \ldots, e_{k}, \ldots, e_{n}\right)$ such that every ideal $\mathfrak{g}_{k}$ is spanned by $\left(e_{1}, \ldots, e_{k}\right)$. We denote $\langle$,$\rangle the scalar product in \mathfrak{g}$ defined by setting $\left\langle e_{i}, e_{j}\right\rangle$ $=\delta_{i j}$ (Kronecker symbol). We set $I=\{k+1, \ldots, n\}$. It is clear that for every $\alpha \in I$ the linear map $D_{\alpha}=a_{\left.d e\right|_{\mathfrak{g}_{k}}}$ lies in $\mathscr{D}_{F}^{\circ}\left(\mathfrak{g}_{k}\right)$, where $F\left(\mathfrak{g}_{k}\right)$ is the filtration that $F(\mathfrak{g})$ induces in $\mathfrak{g}_{k}$. We define $\left(\xi_{\alpha \beta}, \varepsilon_{\alpha \beta}^{\gamma}\right)$ by putting

$$
\begin{gathered}
\xi_{\alpha \beta}=\sum_{t=1}^{k}\left\langle\left[e_{\alpha}, e_{\beta}\right], e_{t}\right\rangle e_{t}, \\
\varepsilon_{\alpha \beta}^{\gamma}=\left\langle\left[e_{\alpha}, e_{\beta}\right], e_{\gamma}\right\rangle,
\end{gathered}
$$

for $(\alpha, \beta, \gamma) \in I^{3}$. Let us choose a subset $J \subset I$ such that $(\gamma, \beta) \in J \times I$, $\beta \leq \gamma \Rightarrow \beta \in J$. Then the data $K_{J, I}^{F}\left(\mathfrak{g}_{k}\right)=\left\{D_{\alpha} \xi_{\alpha \beta}, \varepsilon_{\alpha \beta}^{\gamma}\right\}$ are connected by the conditions $\left(c_{\alpha \beta}\right),\left(c_{\alpha \beta \gamma}\right)$ and $\left(c_{J \gamma_{0}}\right)$ of Definition 2.1. The truncated Lie algebra $\left(\mathfrak{g}, K_{J, I}^{F}(\mathfrak{g})\right)$ is a Lie algebra if and only if $J=I$, for the condition $(c \alpha \beta \gamma) \cup\left\{\left(c_{J, \gamma^{\circ}}\right)_{\alpha \beta \gamma}\right\}$ is the Jacobi identity.

2.1. Truncated affine structures in truncated Lie algebras. Let $\left(\mathfrak{g}, K_{J, I}^{F}(\mathfrak{g})\right)$ be a truncated Lie algebra. We suppose that $\mathfrak{g}$ has a Koszul-Vinberg structure $\rho: \mathfrak{g} \rightarrow \mathfrak{g} l(\mathfrak{g})$. We are concerned with data

$$
\pi_{J, I}^{F}(\mathfrak{g})=\left\{A_{\alpha}, U_{\alpha \beta}, v_{\alpha \beta}^{\gamma} \mid(\alpha, \beta, \gamma) \in I^{3}\right\}
$$

where $\left(A_{\alpha}, U_{\alpha \beta}, v_{\alpha \beta}^{\gamma}\right) \in E_{F}^{\circ}(\mathfrak{g}) \times \mathfrak{g} \times \mathbf{R}$ for every $(\alpha, \beta, \gamma) \in I^{3}$.

Definition 2.2. The triple $\left(\mathfrak{g}, \rho, \pi_{J, I}^{F}(\mathfrak{g})\right)$ is a truncated Koszul-Vinberg structure in the truncated Lie algebra $\left(\mathfrak{g}, K_{J, I}^{F}(\mathfrak{g})\right)$ if the data $\pi_{J, I}^{F}(\mathfrak{g})$ and $K_{J, I}^{F}(\mathfrak{g})$ are connected by the following conditions:

$$
\begin{aligned}
& \left(k_{\alpha}\right):\left[A_{\alpha}, \rho(X)\right]=\rho\left(D_{\alpha} X\right) \text { for every }(\alpha, X) \in I \times \mathfrak{g}, \\
& \left(k_{\alpha \beta}\right):\left(A_{\alpha}\left(A_{\beta}-D_{\beta}\right)-\left(A_{\beta}-D_{\eta}\right) D_{\alpha}-\sum_{t} v_{\alpha \beta}^{t}\left(A_{t}-D_{t}\right)\right) \cdot X=\rho(X) \cdot U_{\alpha \beta} \text { for } \\
& (\alpha, \beta, X) \in I^{2} \times \mathfrak{g}, \\
& \left(k_{\alpha \beta \gamma}\right): A_{\alpha} U_{\beta \gamma}-\left(A_{\gamma}-D_{\gamma}\right) U_{\alpha \beta}+\sum_{t}\left(v_{\beta \gamma}^{t} U_{\alpha t}-v_{\alpha \beta}^{t} U_{t \gamma}\right) \\
& \quad=A_{\beta} U_{\alpha \gamma}-\left(A_{\gamma}-D_{\gamma}\right) U_{\beta \alpha}+\sum_{t}\left(v_{\alpha \gamma}^{t} U_{\beta t}-v_{\beta \alpha}^{t} U_{t \gamma}\right) \quad \text { for every }(\alpha, \beta, \gamma) \in I^{3},
\end{aligned}
$$


$\left(k_{J \gamma_{0}}\right) \alpha \beta \gamma: \sum_{t}\left(v_{\beta \gamma}^{t} v_{\alpha t}^{\gamma_{0}}-v_{\alpha \gamma}^{t} v_{\beta t}^{\gamma_{0}}-\varepsilon_{\alpha \beta}^{t} v_{t \gamma}^{\gamma_{0}}\right)=0 \quad$ for every $\left(\gamma_{0}, \alpha, \beta, \gamma\right) \in J \times I^{3}$; moreover $\left(\xi_{\alpha \beta}, \varepsilon_{\alpha \beta}^{\gamma}\right)$ and $\left(U_{\alpha \beta}, v_{\alpha \beta}^{\gamma}\right)$ are connected by

$$
\begin{gathered}
U_{\alpha \beta}-U_{\beta \alpha}-\xi_{\alpha \beta}=0, \quad v_{\alpha \beta}^{\gamma}-v_{\beta \alpha}^{\gamma}-\varepsilon_{\alpha \beta}^{\gamma}=0, \\
v_{\alpha \beta}^{\gamma}=0 \quad \text { if } \gamma \geq \min (\alpha, \beta) .
\end{gathered}
$$

Remark. We already observed that $\left(\mathfrak{g}, K_{J, I}^{F}(\mathfrak{g})\right)$ is a Lie algebra if and only if $J=I$. Therefore the truncated Koszul-Vinberg $\left(\mathfrak{g}, \rho, \pi_{J, I}^{F}(\mathfrak{g})\right)$ becomes a Koszul-Vinberg structure in that Lie algebra, so that the sequence $0 \rightarrow \mathfrak{g} \rightarrow$ $\mathfrak{g} \times \mathbf{R}^{\# I} \rightarrow \mathbf{R}^{\# I} \rightarrow 0$ is an exact sequence of Koszul-Vinberg structures. If $\# I>1$, the completeness of $(\mathfrak{g}, \rho)$ will not insure the completeness of $\left(\mathfrak{g}, \rho, \pi_{J, I}^{F}(\mathfrak{g})\right)$.

2.2. The $D$-extension property. Let us examine the particular case where $\# I=1$. Then $K_{J, I}^{F} \mathfrak{g}=\{D\}$ with $D \in \mathscr{D}_{F}^{\circ}(\mathfrak{g})$ and the truncated Lie algebra $\left(\mathfrak{g}, K_{J, I}^{F}(\mathfrak{g})\right)$ is the $(n+1)$-dimensional Lie algebra $\mathfrak{g} \times \mathbf{R}$ with the following bracket operation:

$$
[(X, x),(Y, y)]=([X, Y]+D(x Y-y X), 0) .
$$

Therefore the conditions of Definition 2.2 become

$$
[A, \rho(X)]=\rho(D X) \text { and }\left(A^{2}+D^{2}-2 A D\right) \cdot X=\rho(X) U
$$

for some element $U \in \mathfrak{g}$.

Definition 2.3. Let $\rho \in \mathrm{KV}_{F}(\mathfrak{g})=\{\rho \in \mathrm{KV}(\mathfrak{g}) \mid \rho$ preserves $F(\mathfrak{g})\}$ and let $D \in$ $\mathscr{D}_{F}^{\circ}(\mathfrak{g})$. We say that $\rho$ has the $D$-extension property if we can find $(A, U) \in$ $E_{F}^{\circ}(\mathfrak{g}) \times \mathfrak{g}$ such that the following conditions hold:

$[A, \rho(X)]=\rho(D X)$ and $\left(A^{2}+D^{2}-2 A D\right) \cdot X=\rho(X) U$ for every $X \in \mathfrak{g}$.

Let $D \in \mathscr{D}_{F}^{\circ}(\mathfrak{g})$. We denote by $\mathfrak{g}_{D}$ the Lie algebra $\mathfrak{g} \times R$ with the bracket operation, $[(X, x),(Y, y)]=([X, Y]+D(x y-y X), 0)$. The flag $F(\mathfrak{g})$ extends to the flag of ideals $F(\mathfrak{g})=\mathfrak{g}_{D} \supset \mathfrak{g} \supset \cdots \supset \mathfrak{g}_{k} \supset \cdots$. Let $\mathrm{KV}_{F}(\mathfrak{g})$ be the set of Koszul-Vinberg structures which preserve $F(\mathfrak{g})$.

Lemma 2.1. Let $\rho \in \mathrm{KV}_{F}(\mathfrak{g})$ and let $D \in \mathscr{D}_{F}^{\circ}(\mathfrak{g})$. Suppose that $\rho$ has the D-extensions property, and let $A \in E_{F}^{\circ}(\mathfrak{g})$ be such that $[A, \rho(X)]=\rho(D X)$ for every $X \in \mathfrak{g}$. Then the linear map $B=A-D: \mathfrak{g} \mapsto \mathfrak{g}$ is a 1-dimensional cocycle with respect to the linear representation $\rho: \mathfrak{g} \rightarrow \mathfrak{g l}(\mathfrak{g})$.

Proof. We have to prove that the identity $\rho(X) B \cdot Y-\rho(Y) B X-B[X, Y]=0$ holds for every $(X, Y) \in \mathfrak{g} \times \mathfrak{g}$. Indeed we have $\rho(X) B Y=\rho(X)(A-D) Y=$ $\rho(X) A Y-\rho(X) D Y$. On the other hand we get $D[X, Y]=[D X, Y]+[X, D Y]$, so that $B[X, Y]=A[X, Y]-[D X, Y]-[X, D Y]$. Since $\rho \in \mathrm{KV}(\mathfrak{g})$, we must write $[X, Y]=\rho(X) \cdot Y-\rho(Y) X$, so that

$$
\begin{gathered}
\rho(X) B Y-\rho(Y) B X-B[X, Y]=\rho(X) A Y-\rho(Y) A X-\rho(X) D+\rho(Y) D X \\
-A \rho(X) Y+A \rho(Y) X+\rho(D X) Y-\rho(Y) D X+\rho(X) D^{-} \rho(D Y) X \\
=[\rho(X), A] Y-[\rho(Y), A] X+\rho(D X) Y-\rho(D Y) X=0 . \quad \text { Q.E.D. }
\end{gathered}
$$


We have the following result:

Proposition 2.1. Let $\rho \in \mathrm{KV}_{F}(\mathfrak{g})$ and let $D \in \mathscr{D}_{F}^{\circ}(\mathfrak{g})$. If $\rho$ has the D-extension property, then $\rho$ extends to some $\rho_{D} \in \mathrm{KV}_{F}\left(\mathfrak{g}_{D}\right)$. Moreover $\rho_{D}$ is complete if and only if $\rho$ is complete.

Proof. Let $(A, U) \in E_{F}^{\circ}(\mathfrak{g}) / \mathfrak{g}$ be such that the following identities hold:

$$
[A, \rho(X)]=\rho(D X) \text { and }\left(A^{2}+D^{2}-2 A D\right) \cdot X=\rho(X) U \quad \text { for every } X \in \mathfrak{g} .
$$

Let us define the linear map $\rho_{D}: \mathfrak{g}_{D} \rightarrow \mathfrak{g l}\left(\mathfrak{g}_{D}\right)$ by setting

$$
\rho_{D}(X, x) \cdot(Y, y)=((\rho(X)+x A) Y+y((A-D) X+x U), 0) .
$$

Then we have

$$
\begin{aligned}
& \rho_{D}(X, x)(Y, y)-\rho_{D}(Y, y)(X, x) \\
&= \rho(X) Y+x A Y+y((A-D) X+x U) \\
&-\rho(Y) X+y A X+x((A-D) Y+y U) \\
&= \rho(X) Y-\rho(Y) X+D(x Y-y X) \\
&= {[(X, x),(Y, y)] . }
\end{aligned}
$$

Thus it remains to prove that $\rho_{D}$ is a linear representation. We have

$$
\begin{aligned}
& \rho_{D}(X, x) \rho_{D}(Y, y) \cdot(Z, z) \\
&= \rho(X)+x A((\rho(Y)+y A) Z+z((A-D) Y+y U)) \\
&=(\rho(X) \rho(Y)+x A \rho(Y)+y \rho(X) A) Z \\
&+z(\rho(X)+x A)((A-D) Y+y U)
\end{aligned}
$$

so that by antisymmetrizing with respect to the pair $((X, x),(Y, y))$ we obtain

$$
\begin{aligned}
{\left[\rho_{D}(X, x), \rho_{D}(Y, y)\right](Z, z)=([\rho(X), \rho(Y)]+[A, \rho(x Y-y X)]) Z } \\
\quad+z((\rho(X)+x A)((A-D) Y+y U)-(\rho(Y)+y A)((A-D) X+x U)) \\
=([\rho(X), \rho(Y)]+\rho(D(x Y-y X)) Z \\
\quad+z(\rho(X) B Y-\rho(Y) B X-\rho(x Y-y X) U-A(A-D)(x Y-y X)) .
\end{aligned}
$$

By Lemma 2.1 and Definition 2.3 we get

$$
\begin{aligned}
{\left[\rho_{D}(X, x), \rho_{D}(Y, y)\right](Z, z)=} & \rho([X, Y]+D(x Y-y X)) Z \\
& +z(A-D)([X, Y]+D(x Y-y X)) \\
= & \rho_{D}([(X, x),(Y, y)])(Z, z) .
\end{aligned}
$$

Now let us suppose that $\rho$ is complete. Then every linear map $\rho(X)$ is a nilpotent endomorphism of the vector space $\mathfrak{g},[1,10]$. Since $\rho$ belongs to $\mathrm{KV}_{F}(\mathfrak{g})$ we can choose a basis $\left(e_{1}, \ldots, e_{n}\right)$ in $\mathfrak{g}$ such that every ideal $\mathfrak{g}_{k}$ of the flag $F(g)$ is spanned by $\left(e_{1}, \ldots, e_{k}\right)$. Then the matrices associated to $\rho(X)$, 
to $D$ and to $A$, respectively, are upper triangular i.e. they are of the following form

$$
\left[\begin{array}{llll}
0 & & & \\
& \cdot & & \\
0 & & \cdot & \\
& & & 0
\end{array}\right]
$$

Let us therefore fix $\left(Y_{0}, y_{0}\right) \in \mathfrak{g}_{D}$ and consider the linear map $\psi_{Y_{0}, y_{0}}:(X, x)$ $\rightarrow \rho_{D}(X, x)\left(Y_{0}, y_{0}\right)+(X, x)$. If $(X, x)$ lies in the kernel of $\psi_{Y_{0}, y_{0}}$ we have the following system:

$$
\left\{\begin{array}{l}
(\rho(X)+x A) Y_{0}+y_{0}((A-D) X+x U)+X=0 \\
x=0
\end{array}\right.
$$

so that $(X, x)=(X, 0)$ and our system is reduced to the equation

$$
\rho(X) Y_{0}+y_{0}(A-D) X+X=0 .
$$

On the other hand $\rho(X), D$ and $A$ have the form $(* *)$ in the basis $\left(e_{1}, \ldots\right.$, $\left.e_{n}\right)$; therefore the linear map $X \rightarrow \rho(X) Y_{0}+y_{0}(A-D) X$ is nilpotent, so that the condition $\rho(X) Y_{0}+y_{0}(A-D) X+X=0$ is equivalent to $X=0$. This ends the proof of Proposition 2.1.

Examples of $\rho$ with $D$-extension properties. (1) Let $\mathfrak{g}$ be the commutative Lie algebra $\mathbf{R}^{n}$ and let $\left(\mathbf{R}^{n}, 0\right)$ be the trivial Koszul-Vinberg structure in $\mathbf{R}^{n}$. Then $\left(\mathbf{R}^{n}, 0\right)$ has the $D$-extension property for every $D \in \operatorname{End}\left(\mathbf{R}^{n}\right)$. Indeed it suffices to take $A=D$.

(2) Let $\mathfrak{h}_{3}$ be the 3-dimensional Heisenberg algebra. Let $\left(e_{1}, e_{2}, e_{3}\right)$ be a basis of $\mathfrak{h}_{3}$ such that $\left[e_{2}, e_{3}\right]=e_{1},\left[e_{1}, e_{2}\right]=\left[e_{1}, e_{3}\right]=0$. We consider the flag $F\left(\mathfrak{h}_{3}\right)$ spanned by the basis $\left(e_{1}, e_{2}, e_{3}\right) ; F\left(\mathfrak{h}_{3}\right)$ is a flag of ideals. Let $D$ be the element of $\mathscr{D}_{F}^{\circ}\left(\mathfrak{h}_{3}\right)$ whose matrix in $\left(e_{1}, e_{2}, e_{3}\right)$ is the following:

$$
D=\left[\begin{array}{lll}
0 & 1 & 1 \\
0 & 0 & 1 \\
0 & 0 & 0
\end{array}\right] \text {. }
$$

We consider the linear map $\rho: \mathfrak{h}_{3} \rightarrow E_{F}^{\circ}\left(\mathfrak{h}_{3}\right)$ which is given by

$$
\rho\left(x_{1}, x_{2}, x_{3}\right)\left(y_{1}, y_{2}, y_{3}\right)=\left(\left(a x_{2}+x_{3}\right) y_{2}+2 x_{2} y_{3}, 0,0\right) \text {. }
$$

The matrix of $\rho\left(x_{1}, x_{2}, x_{3}\right)$ in the basis $\left(e_{1}, e_{2}, e_{3}\right)$ is

$$
\rho\left(x_{1}, x_{2}, x_{3}\right)=\left[\begin{array}{ccc}
0 & a x_{2}+x_{3} & 2 x_{2} \\
0 & 0 & 0 \\
0 & 0 & 0
\end{array}\right] .
$$

We get $\rho\left(x_{1}, x_{2}, x_{3}\right)\left(y_{1}, y_{2}, y_{3}\right)-\rho\left(y_{1}, y_{2}, y_{3}\right)\left(x_{1}, x_{2}, x_{3}\right)=\left(x_{2} y_{3}-x_{3} y_{2}, 0,0\right)$ $=\left[\left(x_{1}, x_{2}, x_{3}\right),\left(y_{1}, y_{2}, y_{3}\right)\right]$. On the other hand one see that

$$
\rho\left(\left[\left(x_{1}, x_{2}, x_{3}\right),\left(y_{1}, y_{2}, y_{3}\right)\right]\right)=\left[\rho\left(x_{1}, x_{2}, x_{3}\right), \rho\left(y_{1}, y_{2}, y_{3}\right)\right]=0 \text {. }
$$

Let us look for an element $A \in E_{F}^{\circ}\left(\mathfrak{h}_{3}\right)$ such that

$$
\left[A, \rho\left(x_{1}, x_{2}, x_{3}\right)\right]=\rho\left(D\left(x_{1}, x_{2}, x_{3}\right)\right) \text {. }
$$


We set

$$
A=\left[\begin{array}{lll}
0 & \alpha & \beta \\
0 & 0 & \gamma \\
0 & 0 & 0
\end{array}\right]
$$

so that

$$
\left[A, \rho\left(x_{1}, x_{2}, x_{3}\right)\right]=\left[\begin{array}{ccc}
0 & 0 & -\gamma\left(a x_{2}+x_{3}\right) \\
0 & 0 & 0 \\
0 & 0 & 0
\end{array}\right]
$$

and

$$
\rho(D(x))=\rho\left(x_{2}+x_{3}, x_{3}, 0\right)=\left[\begin{array}{ccc}
0 & a x_{3} & 2 x_{3} \\
0 & 0 & 0 \\
0 & 0 & 0
\end{array}\right] .
$$

Thus, if $a \neq 0$ there is no $A \in E_{F}^{\circ}\left(\mathfrak{h}_{3}\right)$ such that $[A, \rho(X)]=\rho(D X)$. This proves that the element $\rho_{0} \in \mathrm{KV}_{F}\left(\mathfrak{h}_{3}\right)$ given by

$$
\rho_{0}\left(x_{1}, x_{2}, x_{3}\right)=\left[\begin{array}{ccc}
0 & x_{2} & x_{2} \\
0 & 0 & 0 \\
0 & 0 & 0
\end{array}\right]
$$

does not have the $D$-extension property where

$$
D=\left[\begin{array}{lll}
0 & 1 & 1 \\
0 & 0 & 1 \\
0 & 0 & 0
\end{array}\right]
$$

\section{NORMAL AFFINE STRUCTURES}

Let $\mathfrak{g}$ be a nilpotent Lie algebra with the complete Koszul-Vinberg structure $\rho: \mathfrak{g} \rightarrow \mathfrak{g} l(\mathfrak{g})$. Since $\rho$ is compete there is a $\rho$-preserved flag $F(\mathfrak{g})=\mathfrak{g} \supset \cdots \supset$ $\mathfrak{g}_{k} \supset \cdots,[9,10]$. The flat $F(\mathfrak{g})$ is not necessarily a flag of ideals. We set $n=\operatorname{dim} \mathfrak{g}$.

Definition 3.1. A complete Koszul-Vinberg structure $\rho$ is called normal structure if it has the following properties:

(i) there is a $\rho$-preserved flag of ideals $F(\mathfrak{g})=\mathfrak{g} \supset \cdots \supset \mathfrak{g}_{k} \supset \cdots$ which is finer than the lower central series $\mathfrak{g} \supset C_{1}(\mathfrak{g}) \supset \cdots \supset C_{k}(\mathfrak{g}) \supset \cdots$,

(ii) if $(k, l)$ is a pair of integers with $k \leq l$ and $(k, l) \in\{1, \ldots, n\}^{2}$, then we have $\left[\mathfrak{g}_{l}, \mathfrak{g}_{k}\right]=\rho\left(\mathfrak{g}_{l}\right) \mathfrak{g}_{k}$.

Remark 3.1. Let $\mathfrak{g}$ be a nilpotent Lie algebra; let $f(\mathfrak{g})=\mathfrak{g} \supset \cdots \supset \mathfrak{g}_{k+1} \supset$ $\mathfrak{g}_{k} \supset \ldots$ be a flag of ideals which is finer than the lower central series of $\mathfrak{g}$. Let $\mathrm{KV}_{F(\mathfrak{g})}^{n}$ be the set of normal Koszul-Vinberg structures $\rho: \mathfrak{g} \rightarrow \mathfrak{g l}(\mathfrak{g})$ such that the couple $(F(g), \rho)$ satisfies (i) and (ii) of Definition 3.1. For every ideal $\mathfrak{g}_{k+1}$ taken in the sequence $F(\mathfrak{g})$, let $\rho_{k+1}=\rho_{\mid \mathfrak{g}_{k+1}} \rightarrow \mathfrak{g} l\left(\mathfrak{g}_{k+1}\right)$ and $F\left(\mathfrak{g}_{k+1}\right)=$ $\mathfrak{g}_{k+1} \supset \mathfrak{g}_{k} \supset \cdots$. Then the couple $\left(F\left(\mathfrak{g}_{k+1}\right), \rho_{k+1}\right)$ satisfies conditions (i) and (ii). Hence, $\rho_{k+1}$ is also a normal Koszul-Vinberg structure in $\mathfrak{g}_{k+1}$. 
Examples of normal structures. 1. Let $\mathfrak{g}$ be the commutative Lie algebra $\mathbf{R}^{n}$. By virtue of (ii), the unique normal structure in $\mathbf{R}^{n}$ is a trivial one $\left(\mathbf{R}^{n}, \rho=0\right)$.

2. Let $\mathfrak{h}_{3}$ be the 3-dimensional Heisenberg algebra. We take a basis $\left(e_{1}, e_{2}\right.$, $\left.e_{3}\right)$ in $\mathfrak{h}_{3}$ such that $\left[e_{2}, e_{3}\right]=e_{1},\left[e_{2}, e_{1}\right]=\left[e_{3}, e_{1}\right]=0$. We consider the flag $F(\mathfrak{g})$ which is spanned by $\left(e_{1}, e_{2}, e_{3}\right)$; it is a flag of ideals. We construct the linear map $\rho: \mathfrak{h}_{3} \rightarrow \mathfrak{g l}\left(\mathfrak{h}_{3}\right)$ by setting

$$
\rho\left(x_{1}, x_{2}, x_{3}\right)\left(y_{1}, y_{2}, y_{3}\right)=\left(a x_{3} y_{2}+(1+a) x_{2} y_{3}, 0,0\right) .
$$

The matrix of $\rho\left(x_{1}, x_{2}, x_{3}\right)$ in $\left(e_{1}, e_{2}, e_{3}\right)$ is

$$
\rho\left(x_{1}, x_{2}, x_{3}\right)=\left[\begin{array}{ccc}
0 & a x_{3} & (1+a) x_{2} \\
0 & 0 & 0 \\
0 & 0 & 0
\end{array}\right] \text {. }
$$

One sees that $\rho$ has properties (i) and (ii) of Definition 3.1, that is $\rho$ is normal if and only if $(1+a) a \neq 0$. Every complete normal Koszul-Vinberg structure in $\mathfrak{h}_{3}$ has this form.

Remark 3.2. Let $\mathfrak{g}$ be a nilpotent Lie algebra. We denote by $\mathrm{KV}^{\mathcal{c}}(\mathfrak{g})$ the set of complete Koszul-Vinberg structures $(\mathfrak{g}, \rho)$. L. Auslander conjectured that for every element $(\mathfrak{g}, \rho) \in \mathrm{KV}^{c}(\mathfrak{g})$ the linear representation $\rho$ is not injective. It is David Fried who constructed a counterexample to this conjecture. By the virtue of Definition 3.1 every normal structure $(\mathfrak{g}, \rho)$ has nontrivial translations. A paper of $\mathrm{H}$. Kim contains a complete classification of 4-dimensional complete Koszul-Vinberg structures without nontrivial translation $(\mathrm{H}$. Kim, Complete left invariant affine structure on nilpotent Lie groups, J. Differential Geom. 24 (1986)).

Let us fix a normal Koszul-Vinberg structure in the nilpotent Lie algebra $\mathfrak{g}$. We fix some flag $F(\mathfrak{g})=\mathfrak{g} \supset \cdots \supset \mathfrak{g}_{k} \supset \cdots$ and some basis $\left(e_{1}, \ldots, e_{n}\right)$ which satisfy the conditions of Definition 3.1. Let $D \in \mathscr{D}_{F}^{\circ}(\mathfrak{g})$. We consider the Lie algebra $\mathfrak{g}_{D}$ as in Proposition 2.1 and we take the flag $F\left(\mathfrak{g}_{D}\right)=\mathfrak{g}_{D} \supset \mathfrak{g} \supset \cdots \supset$ $\mathfrak{g}_{k} \supset \cdots$. We set $\overline{\mathfrak{g}}_{D}=\mathfrak{g}_{D} / \mathfrak{g}_{1}$ and $\overline{\mathfrak{g}}=\mathfrak{g} / \mathfrak{g}_{1}$, so that the following diagram is commutative:

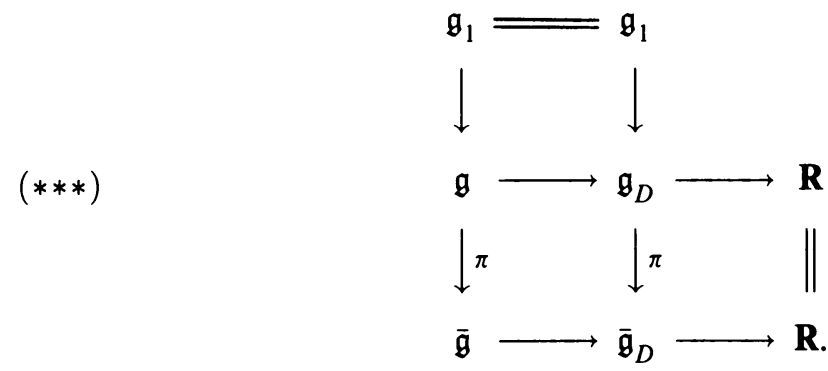

Let us suppose that $\rho$ has the $D$-extension property. Then by virtue of Proposition 2.1, $\rho$ can be extended to some Koszul-Vinberg structure $\rho_{D}$ in $\mathfrak{g}_{D}$ such that the flag $F\left(\mathfrak{g}_{D}\right)$ is $\rho_{D}$-preserved. Therefore $(* * *)$ becomes a diagram 
of Koszul-Vinberg structures

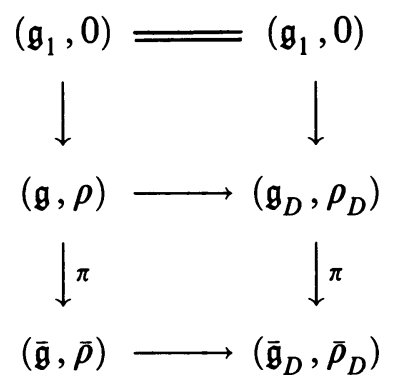

where $\bar{\rho}\left(\right.$ resp. $\left.\bar{\rho}_{D}\right)$ is the quotient structure of $\rho$ (resp. $\left.\rho_{D}\right)$. We shall prove the following theorem.

Theorem 3.1. Keeping the previous notations, let us suppose that $\rho$ and $\bar{\rho}_{D}$ are normal and that for every $n$-dimensional ideal $\mathfrak{a} \subset \mathfrak{g}_{D}$ such that $\mathfrak{g}_{n-1} \subset \mathfrak{a}$, $\rho_{\mathfrak{a}}=\rho_{D / \mathfrak{a}}$ is normal. Then $p$ has an extended structure $\tilde{\rho}_{D}$ which is normal and $\overline{\tilde{\rho}}_{D}=\tilde{\rho}_{D}$.

Proof. Since $\rho$ and $\bar{\rho}_{D}$ are normal, the structure $\bar{\rho}$ is normal also. Thus we have to prove that the diagram

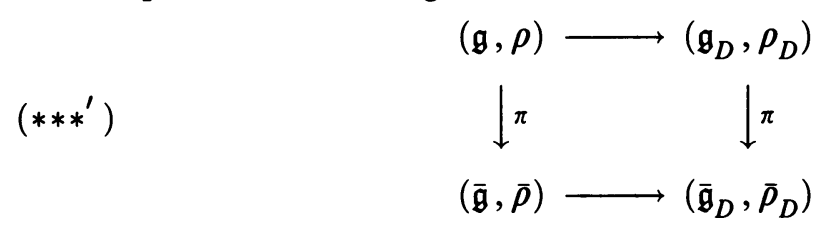

is a commutative diagram of normal structures. We begin by fixing a basis $\left(e_{1}, \ldots, e_{n+1}\right)$ of $g_{D}$ such that $\left(e_{1}, \ldots, e_{n}\right)$ (resp. $\left(\bar{e}_{2}, \ldots, \bar{e}_{n+1}\right)=\left(\pi e_{2}, \ldots\right.$, $\left.\pi e_{n+1}\right)$ ) spans the flag $F(\mathfrak{g})$ (resp. $F\left(\overline{\mathfrak{g}}_{D}\right)$ ). We suppose that $F(\mathfrak{g})$ (resp. $F\left(\overline{\mathfrak{g}}_{D}\right)$ ) satisfies (i) and (ii) of Definition 3.1. Let $\omega$ be the 2-dimensional scalar cocycle of $\overline{\mathfrak{g}}_{D}$ such that the bracket in $\mathfrak{g}_{D}$ is given by setting

$$
[(x, X),(y, Y)]=(\omega(X, Y),[X, Y]) \text {. }
$$

Then $\bar{\rho}_{D}$ has the $[\omega]$-lifting property, so that we can find $\varphi \in C^{1}\left(\overline{\mathfrak{g}}_{D}, \overline{\mathfrak{g}}_{D}^{*}\right)$ such that $\rho_{D}$ is given as

$$
\rho_{D}(x, X) \cdot(y, Y)=\left(\varphi(X, Y), \bar{\rho}_{D}(X) Y\right) .
$$

We have to prove that the flag $F\left(\mathfrak{g}_{D}\right)=\mathfrak{g}_{D} \supset F(\mathfrak{g})$ has properties (i) and (ii) of Definition 3.1 for some $\tilde{\rho}_{D}$. It is easy to see that condition (i) holds. Let us prove that condition (ii) also holds. It is assumed that condition (ii) holds for $F\left(\overline{\mathfrak{g}}_{D}\right)$ and for every $n$-dimensional ideal $\mathfrak{a} \subset \mathfrak{g}_{D}$ with $\mathfrak{g}_{n-1} \subset \mathfrak{a}$, (for $\rho_{\mathfrak{a}}=\rho_{D \mid \mathfrak{a}}$ is normal); so that is sufficient to prove that for every ideal $\mathfrak{g}_{k}$ we have

$$
\left[\mathfrak{g}_{D}, \mathfrak{g}_{k}\right]=\rho_{D}\left(\mathfrak{g}_{D}\right) \mathfrak{g}_{k} .
$$

Let $\mathfrak{a}$ be the $n$-dimensional ideal of $\mathfrak{g}_{D}$ spanned by $e_{1}, \ldots, e_{n-1}, e_{n+1}$, then $\mathfrak{a}=\mathfrak{g}_{n-1} \oplus \mathbf{R} e_{n+1}$. By the virtue of our hypothesis one has

$$
\left[\mathfrak{g}_{n}=\mathfrak{g}, \mathfrak{g}_{k}\right]=\rho(\mathfrak{g}) \mathfrak{g}_{k} \quad \text { and } \quad\left[\mathfrak{a}, \mathfrak{g}_{k}\right]=\rho(\mathfrak{a}) \mathfrak{g}_{k} .
$$


The above equalities imply $\left[\mathfrak{g}_{D}, \mathfrak{g}_{k}\right]=\rho_{D}\left(\mathfrak{g}_{D}\right) \mathfrak{g}_{k}$. That follows from the following lemma which will be useful in forthcoming work.

Lemma 3.1. Let us consider the normal structures in $\left(* * *^{\prime}\right)$. Let $\left(e_{1}, \ldots, e_{n+1}\right)$ be the fixed basis of $\mathfrak{g}_{D}$ as in the proof of Theorem 3.1. Let $\langle$,$\rangle be the scalar$ product in $\mathfrak{g}_{D}$ given by $\left\langle e_{\alpha}, e_{\beta}\right\rangle=\delta \alpha \beta$. We set $\varepsilon_{\alpha \beta}^{\gamma}=\left\langle\left[e_{\alpha}, e_{\beta}\right], e_{\gamma}\right\rangle$ and $v_{\alpha \beta}^{\gamma}=$ $\left\langle\rho\left(e_{\alpha}\right) e_{\beta}, e_{\gamma}\right\rangle$. We identify $\left(e_{2}, \ldots, e_{n+1}\right)$ with the projected basis $\left(\bar{e}_{n}, \ldots, \bar{e}_{n+1}\right)$ in $\overline{\mathfrak{g}}_{\mathscr{D}}$. We suppose that the following condition holds:

$$
\left(n_{\alpha \beta \gamma}\right): \varepsilon_{\alpha \beta}^{\gamma} \neq 0 \Rightarrow v_{\alpha \beta}^{\gamma}\left(v_{\alpha \beta}^{\gamma}-\varepsilon_{\alpha \beta}^{\gamma}\right) \neq 0
$$

for $\left(\overline{\mathfrak{g}}_{D}, \bar{\rho}_{D}\right)$ and for every $n$-dimensional ideal $\mathfrak{a} \subset \mathfrak{g}_{D}$ with $\mathfrak{g}_{n-1} \subset \mathfrak{a}$. Then we can choose some extended structure $\left(\mathfrak{g}_{D}, \tilde{\rho}_{D}\right)$ such that $\left(n_{\alpha \beta \gamma}\right)$ holds again. Proof. Let us identify $\left(\bar{e}_{2}, \ldots, \bar{e}_{n+1}\right)$ with $\left(e_{2}, \ldots, e_{n+1}\right)$; then if $(\alpha, \beta, \gamma) \in$ $\{2, \ldots, n+1\}^{3}$ we have $\varepsilon_{\alpha \beta}^{\gamma} \neq 0 \Rightarrow\left(v_{\alpha \beta}^{\gamma}\right)\left(v_{\alpha \beta}^{\gamma}-\varepsilon_{\alpha \beta}^{\gamma}\right) \neq 0$. On the other hand the inclusion $\mathfrak{g}_{n-1} \subset \mathfrak{g}$ implies that if $(\alpha, \beta) \in\{2, \ldots, n\}^{2}$ then

$$
\omega\left(w_{\alpha}, e_{\beta}\right) \neq 0 \Rightarrow \varphi\left(e_{\alpha}, e_{\beta}\right)\left(\varphi\left(e_{\alpha}, e_{\beta}\right)-\omega\left(e_{\alpha}, e_{\beta}\right)\right) \neq 0 \text {. }
$$

Let us consider the ideal $\mathfrak{a}$ of $\mathfrak{g}_{D}$ which is spanned by $\left(e_{1}, \ldots, e_{n-1}, e_{n+1}\right)$. It is clear that $\rho_{D}$ induces a Koszul-Vinberg structure $\rho_{\mathrm{a}}$ in that ideal and that structure is normal. Then, for every pair $(\alpha, \beta) \in\{2, \ldots, n+1\}^{2}$ such that $(\alpha, \beta) \neq(n, n+1)$, one has

$$
\omega\left(e_{\alpha}, e_{\beta}\right) \neq 0 \Rightarrow \varphi\left(e_{\alpha}, e_{\beta}\right)\left(\varphi\left(e_{\alpha}, e_{\beta}\right)-\omega\left(e_{\alpha}, e_{\beta}\right)\right) \neq 0 .
$$

Finally we have to prove that by perturbing $\varphi$ we can write

$$
\omega\left(e_{n}, e_{n+1}\right) \neq 0 \Rightarrow \varphi\left(e_{n}, e_{n+1}\right)\left(\varphi\left(e_{n}, e_{n+1}\right)-\omega\left(e_{n}, e_{n+1}\right)\right) \neq 0 \text {. }
$$

Let $\lambda$ be a real number, $\lambda \neq 0$. We define the symmetric bilinear form $S_{\lambda}: \overline{\mathfrak{g}}_{D} \times$ $\overline{\mathfrak{g}}_{D} \rightarrow \mathbf{R}$ by setting

$$
S_{\lambda}\left(e_{\alpha}, e_{\beta}\right)=\lambda\left(\delta_{\alpha n} \delta_{\beta n+1}+\delta_{\beta n} \delta_{\alpha n+1}\right) \text {, }
$$

where $(\alpha, \beta) \in\{2, \ldots, n+1\}^{2}$ and $\delta_{\alpha n}$ is the Kronecker symbol. If $(\alpha, \beta, \gamma)$ $\in\{2, \ldots, n+1\}^{3}$, we compute

$$
d_{\bar{\rho}_{D}} S_{\lambda}\left(e_{\alpha}, e_{\beta}, e_{\gamma}\right)=S_{\lambda}\left(e_{\alpha}, \bar{\rho}_{D}\left(e_{\beta}\right) e_{\gamma}\right)-S_{\lambda}\left(e_{\beta}, \bar{\rho}_{D}\left(e_{\alpha}\right) e_{\gamma}\right)-S_{\lambda}\left(\left[e_{\alpha}, e_{\beta}\right], e_{\gamma}\right) .
$$

Since $\bar{\rho}_{D}$ is normal we have $\bar{\rho}_{D}\left(\overline{\mathfrak{g}}_{D}\right) \overline{\mathfrak{g}}_{D}=\left[\overline{\mathfrak{g}}_{D}, \overline{\mathfrak{g}}_{D}\right] \subset \overline{\mathfrak{g}}_{n-1}$, so that $d_{\bar{\rho}_{D}} S_{\lambda}=0$. We consider the map

$$
\tilde{\varphi}_{\lambda}(X, Y)=\varphi(X, Y)+S_{\lambda}(X, Y) .
$$

Then we have

$$
\tilde{\varphi}_{\lambda}(X, Y)-\tilde{\varphi}_{\lambda}(Y, X)=\omega(X, Y), \quad d_{\bar{\rho}_{D}} \tilde{\varphi}_{\lambda}=0 .
$$

If $(\alpha, \beta) \neq(n, n+1)$ we have $\tilde{\varphi}\left(e_{\alpha}, e_{\beta}\right)=\varphi\left(e_{\alpha}, e_{\beta}\right)$ and $\tilde{\varphi}_{\lambda}\left(e_{n}, e_{n+1}\right)=$ $\varphi\left(e_{n}, e_{n+1}\right)+\lambda$; thus we can choose the scalar $\lambda$ such that

$$
\omega\left(e_{\alpha}, e_{\beta}\right) \neq 0 \Rightarrow \tilde{\varphi}\left(e_{\alpha}, e_{\beta}\right)\left(\tilde{\varphi}_{\lambda}\left(e_{\alpha}, e_{\beta}\right)-\omega\left(e_{\alpha}, e_{\beta}\right)\right) \neq 0
$$


for every $(\alpha, \beta) \in\{2, \ldots, n+1\}^{2}$. Therefore we consider Koszul-Vinberg structure $\tilde{\rho}_{D}$ which is given by

$$
\tilde{\rho}_{D}(x, X)(y, Y)=\left(\tilde{\varphi}_{\lambda}(X, Y), \bar{\rho}_{D}(X) Y\right) .
$$

We see that $\tilde{\rho}_{D}$ is normal and has the following properties:

(i) $\tilde{\rho}_{D}(X) Y=\rho(X) Y$ if $(X, Y) \in \mathfrak{g}$;

(ii) $\hat{\tilde{\rho}}_{D}=\bar{\rho}_{D}$;

so that $\tilde{\rho}_{D}$ is the required Koszul-Vinberg structure.

\section{THE LIFTING PROBLEM FOR NORMAL STRUCTURES}

We fix a $n$-dimensional nilpotent Lie algebra $\mathfrak{g}$ with a fixed complete normal structure $\rho$. Let $F(\mathfrak{g})=\mathfrak{g} \supset \mathfrak{g}_{n-1} \supset \cdots$ be a $\rho$-preserved flag of ideals and we consider a basis $\left(e_{1}, \ldots, e_{n}\right)$ which spans $F(\mathfrak{g})$. We assume that $F(\mathfrak{g})$ and $\left(e_{1}, \ldots, e_{n}\right)$ satisfy conditions (i) and (ii) of Definition 3.1.

Let $\mathfrak{g}_{n-3}$ be the $(n-3)$-dimensional ideal of the flag $F(\mathfrak{g})$. We set $\mathfrak{a}_{3}=$ $\mathfrak{g} / \mathfrak{g}_{n-3}$. Then $\mathfrak{a}_{3}$ is either the Heisenberg algebra $\mathfrak{h}_{3}$ or the commutative Lie algebra $\mathbf{R}^{3}$. We identify $\left(e_{n-2}, e_{n-1}, e_{n}\right)$ with a basis $\left(\bar{e}_{1}, \bar{e}_{2}, \bar{e}_{3}\right)$ of $\mathfrak{a}_{3}$.

Let us set $I=(n-2, n-1, n) \cong(1,2,3)$ and let $\rho_{0}$ be the Koszul-Vinberg structure of $\mathfrak{g}_{n-3}$ which is induced by $\rho$. Let $\langle$,$\rangle be the Euclidean product in$ $\mathfrak{g}$ given by $\left\langle e_{\alpha}, e_{\beta}\right\rangle=\delta_{\alpha \beta}$. We set

$$
\begin{aligned}
D_{\alpha} & =\operatorname{ad}\left(\bar{e}_{\alpha}\right)_{\mid g_{n-3}} ; \\
\xi_{\alpha \beta} & =\sum_{t=1}^{n-3}\left\langle\left[\bar{e}_{\alpha}, \bar{e}_{\beta}\right], e_{t}\right\rangle e_{t} ; \\
\varepsilon_{\alpha \beta}^{\gamma} & =\left\langle\left[\bar{e}_{\alpha}, \bar{e}_{\beta}\right], \bar{e}_{\gamma}\right\rangle
\end{aligned}
$$

for $(\alpha, \beta, \gamma) \in\{1,2,3\}^{3}$. Then the Lie algebra $\mathfrak{g}$ can be identified with truncated Lie algebra $\left(\mathfrak{g}_{n-3}, K_{I, I}^{F}\left(\mathfrak{g}_{n-1}\right)\right)$ where

$$
K_{I, I}^{F}\left(\mathfrak{g}_{n-3}\right)=\left\{D_{\alpha}, \xi_{\alpha \beta}, \varepsilon_{\alpha \beta}^{\gamma} \mid(\alpha, \beta, \gamma) \in I^{3}\right\} .
$$

On the other hand let us set

$$
\begin{aligned}
A_{\alpha} & =\rho_{n}\left(e_{\alpha}\right)_{\mid g_{n-3}}, \\
U_{\alpha \beta} & =\sum_{t=1}^{n-3}\left\langle\rho\left(\bar{e}_{\alpha}\right) \bar{e}_{\beta}, \bar{e}_{t}\right\rangle e_{t}, \\
v_{\alpha \beta}^{\gamma} & =\left\langle\rho\left(\bar{e}_{\alpha}\right) \bar{e}_{\beta}, \bar{e}_{\gamma}\right\rangle .
\end{aligned}
$$

Then the Koszul-Vinberg structure $\rho$ is defined by the truncated structure $\left(\mathfrak{g}_{n-2}, \rho_{0}, \pi_{I, I}^{F}\left(\mathfrak{g}_{n-3}\right)\right)$ where

$$
\pi_{I, I}^{F}\left(\mathfrak{g}_{n-3}\right)=\left\{A_{\alpha}, U_{\alpha \beta}, v_{\alpha \beta}^{\gamma} \mid(\alpha \beta, \gamma) \in I^{3}\right\} .
$$


In other words the data $K_{I, I}^{F}\left(\mathfrak{g}_{n-3}\right)$ and $\pi_{I, I}^{F}\left(\mathfrak{g}_{n-3}\right)$ are connected as follows

$$
\begin{gathered}
\left(k_{\alpha}\right):\left[A_{\alpha}, \rho_{0}(X)\right]=\rho_{0}\left(D_{\alpha} X\right), \quad X \in \mathfrak{g}_{n-3} ; \\
\left(k_{\alpha \beta}:\left(A_{\alpha}\left(A_{\eta}-D_{\beta}\right)-\left(A_{\beta}-D_{\beta}\right) D_{\alpha}-\sum_{t} v_{\alpha \beta}^{t}\left(A_{t}-D_{t}\right)\right) \cdot X=\rho_{0}(X) U_{\alpha \beta},\right. \\
\left(k_{\alpha \beta \gamma}\right): A_{\alpha} U_{\beta \gamma}-\left(A_{\gamma}-D_{\gamma}\right) U_{\alpha \beta}+\sum_{t}\left(v_{\beta \gamma}^{t} U_{\alpha t}-v_{\alpha \beta}^{t} U_{t \gamma}\right) \\
=A_{\beta} U_{\alpha \gamma}-\left(A_{\gamma}-D_{\gamma}\right) U_{\beta \alpha}+\sum_{t}^{t}\left(v_{\alpha \gamma}^{t}\left(v_{\alpha \gamma}^{t} U_{\beta t}-v_{\beta \alpha}^{t} U_{t \gamma}\right) ;\right. \\
\left(k_{I, \gamma_{0}}\right)_{\alpha \beta \gamma}: \sum_{t}\left(v_{\beta \gamma}^{t} v_{\alpha t}^{\gamma_{0}}-v_{\alpha \gamma}^{t} v_{\beta t}^{\gamma_{0}}-\varepsilon_{\alpha \beta}^{t} v_{t \gamma}^{\gamma_{0}}\right)=0 ; \\
U_{\alpha \beta}-U_{\beta \alpha}=\xi_{\alpha \beta}, \quad v_{\alpha \beta}^{\gamma}-v_{\beta \alpha}^{\gamma}=\varepsilon_{\alpha \beta}^{\gamma}, \quad v_{\alpha \beta}^{\beta}=0 \quad \text { if } \gamma \geq \min (\alpha, \beta) .
\end{gathered}
$$

If $\overline{\mathfrak{a}}_{2}$ is some 2-dimensional ideal in $\mathfrak{a}_{3}$, we set $\mathfrak{a}=\tilde{\pi}^{-1}\left(\overline{\mathfrak{a}}_{2}\right)$, so that $\mathfrak{a}$ is $(n-1)$-dimensional ideal in $\mathfrak{g}$ such $\mathfrak{g}_{n-3} \subset \mathfrak{a} ; \tilde{\pi}$ being the projection such that $\mathfrak{g}_{n-3} \rightarrow \mathfrak{g} \stackrel{\tilde{\pi}}{\rightarrow} \mathfrak{a}_{3}$ is an exact sequence.

Let $\omega$ be some 2-dimensional scalar cocycle in $\mathfrak{g}$; we denote $\mathfrak{g}^{\omega}$ the Lie algebra $\mathbf{R} \times \mathfrak{g}$ with the bracket

$$
[(x, X),(y, Y)]=(\omega(X, Y),[X, Y]) .
$$

Let $\pi$ be the canonical homomorphism $\mathfrak{g}^{\omega} \rightarrow \mathfrak{g}$. Let $F\left(\mathfrak{g}^{\omega}\right)$ be the flag $\pi^{-\uparrow}(F(\mathfrak{g}))$. By setting $\mathfrak{g}_{k+1}^{\omega}=\tilde{\pi}^{-\uparrow}\left(\mathfrak{g}_{k}\right)$ we have the commutative diagram

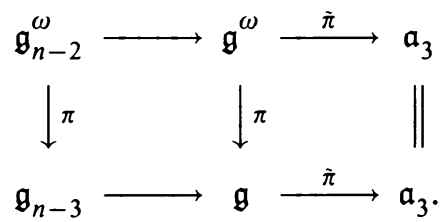

Let $\overline{\mathfrak{a}}_{2}$ be a 2-dimensional ideal in $\mathfrak{a}_{3}$, we set $\mathfrak{a}=\tilde{\pi}^{-1}\left(\overline{\mathfrak{a}}_{2}\right)$. Then $\mathfrak{a}$ is $(n-1)$-dimensional ideal in $\mathfrak{g}$ such that $\mathfrak{g}_{n-3} \subset \mathfrak{a}$. Let us put $\mathfrak{a}^{\omega}=\pi^{-1}(\mathfrak{a})$. The following diagram commutes

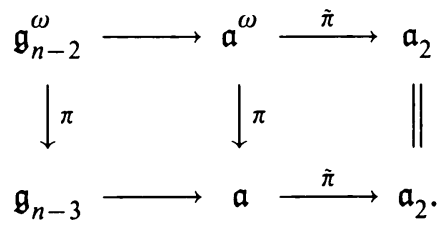

The structure $\rho$ induces the structure $\rho_{\mathfrak{a}}: \mathfrak{a} \rightarrow \mathfrak{g} l(\mathfrak{a})$. If $\left(\overline{\mathfrak{a}}_{2}, \overline{\mathfrak{a}}_{2}^{\prime}\right)$ is a pair of ideals in $\mathfrak{a}_{3}$ we have that

$$
\rho_{\mathfrak{a} \cap \mathfrak{a}^{\prime}}=\rho_{\mathfrak{a} \mid \mathfrak{a} \cap \mathfrak{a}^{\prime}}=\rho_{\mathfrak{a}^{\prime} \mid \mathfrak{a} \cap \mathfrak{a}^{\prime}}
$$


The restriction $\omega_{\mid a \times a}$ is denoted $\omega$ again. If $\rho_{a}$ has the [ $\omega$ ]-lifting property we shall denote by $\rho_{\mathfrak{a}}^{\omega}$ any structure in $\mathfrak{a}^{\omega}$ such that for every $X \in \mathfrak{a}^{\omega}$ we get

$$
\pi \circ \rho_{\mathfrak{a}}^{\omega}(X)=\rho_{\mathfrak{a}}(\pi X) \circ \pi .
$$

Keeping these notations we will prove the following theorem.

Theorem 4.1. Let $(\mathfrak{g}, \rho, \omega)$ be as we just defined. We suppose that the complete normal structure has the following properties:

(i) For every ideal $\overline{\mathfrak{a}}_{2} \subset \mathfrak{a}_{3}, \rho_{\mathfrak{a}}$ has the [ $\left.\omega\right]$-lifting property.

(ii) We can choose the $\rho_{\mathfrak{a}}^{\omega}$ such that given every pair $\left(\overline{\mathfrak{a}}_{2}, \overline{\mathfrak{a}}_{2}^{\prime}\right)$ of ideals, $\rho_{\mathfrak{a}}^{\omega}$ and $\rho_{\mathfrak{a}^{\prime}}^{\omega}$ induce the same structure $\rho_{\mathrm{a} \cap \mathfrak{a}^{\prime}}^{\omega}$ in $\mathfrak{a}^{\omega} \cap \mathfrak{a}^{\prime \omega}$.

(iii) Let $\left(\overline{\mathfrak{a}}_{2}, \overline{\mathfrak{a}}_{2}^{\prime}\right)$ and $\left(\overline{\mathfrak{a}}_{2}^{\prime \prime}, \overline{\mathfrak{a}}_{2}^{\prime \prime \prime}\right)$ be pairs of ideals in $\mathfrak{a}_{3}$, then

$$
\mathfrak{a}^{\omega} \cap \mathfrak{a}^{\prime \omega}=\mathfrak{a}^{\prime \prime \omega} \cap \mathfrak{a}^{\prime \prime \prime \omega} \Rightarrow \rho_{\mathfrak{a} \cap \mathfrak{a}^{\prime}}^{\omega}=\rho_{\mathfrak{a}^{\prime \prime} \cap \mathfrak{a}^{\prime \prime \prime}}^{\omega} .
$$

Then $\rho$ has the $[\omega]$-lifting property.

Proof. Since $\rho$ is normal, its quotient structure $\left(\mathfrak{a}_{3}, \bar{\rho}\right)$ is normal, so that the sequence $0 \rightarrow\left(\mathfrak{g}_{n-3}, \rho_{0}\right) \rightarrow(\mathfrak{g}, \rho) \rightarrow\left(\mathfrak{a}_{3}, \bar{\rho}\right) \rightarrow 0$ is an exact sequence of normal structures.

Since $\mathfrak{a}_{3}$ is either $\mathfrak{h}_{3}$ or $\mathbf{R}^{3}$ we consider the two cases.

Case 1. $a_{3}$ is the commutative Lie algebra $\mathbf{R}^{3}$. In this case the quotient structure $\left(\mathfrak{a}_{3}, \bar{\rho}\right)$ is the trivial structure for it is normal. We consider the ideals

$$
a_{2}=\operatorname{span}\left(\bar{e}_{1}, \bar{e}_{2}\right), \quad a_{2}^{\prime}=\operatorname{span}\left(\bar{e}_{1}, \bar{e}_{3}\right), \quad a_{2}^{\prime \prime}=\operatorname{span}\left(\bar{e}_{2}, \bar{e}_{3}\right) .
$$

Let $\mathfrak{a}, \mathfrak{a}^{\prime}$ and $\mathfrak{a}^{\prime \prime}$ be the corresponding $(n-1)$-dimensional ideals in $\mathfrak{g}$. The flag $F\left(\mathfrak{g}_{n-3}\right)$ is extended to flags

$$
\begin{gathered}
F\left(\mathfrak{g}_{n-3}\right) \subset \tilde{\mathfrak{a}} \subset \mathfrak{a}=F(\mathfrak{a}), \quad F\left(\mathfrak{g}_{n-3}\right) \subset \tilde{\mathfrak{a}}^{\prime} \subset \mathfrak{a}^{\prime}=F\left(\mathfrak{a}^{\prime}\right), \\
F\left(\mathfrak{g}_{n-3}\right) \subset \tilde{\mathfrak{a}}^{\prime \prime \prime} \subset \mathfrak{a}^{\prime \prime}=F\left(\mathfrak{a}^{\prime \prime}\right) .
\end{gathered}
$$

One sees that $F(\mathfrak{a}), F\left(\mathfrak{a}^{\prime}\right)$ and $F\left(\mathfrak{a}^{\prime \prime}\right)$ are spanned by $\left(e_{1}, \ldots, e_{n-3}, e_{n-2}\right.$, $\left.e_{n-1}\right),\left(e_{1}, \ldots, e_{n-3}, e_{n-2}, e_{n}\right)$ and $\left(e_{1}, \ldots, e_{n-3}, e_{n-1}, e_{n}\right)$ respectively. We already saw that $\mathfrak{g}$ is identified with the truncated Lie algebra $\left(\mathfrak{g}_{n-3}, K_{I, I}^{F}\left(\mathfrak{g}_{n-3}\right)\right)$. Since $a_{3}=\mathbf{R}^{3}$ we have

$$
K_{I, I}^{F}\left(\mathfrak{g}_{n-3}\right)=\left\{D_{\alpha}, \xi_{\alpha \beta} \mid(\alpha, \beta) \in I^{2}\right\} .
$$

The subalgebras $\tilde{\mathfrak{a}}, \tilde{\mathfrak{a}}^{\prime}$ and $\tilde{\mathfrak{a}}^{\prime \prime}$ are nothing but $\left(\mathfrak{g}_{n-3}\right)_{D_{1}},\left(\mathfrak{g}_{n-3}\right)_{D_{2}}$ and $\left(\mathfrak{g}_{n-3}\right)_{D_{3}}$ respectively, therefore, the following diagram is commutative

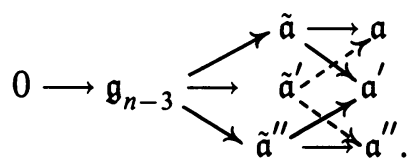

Moreover we have $\tilde{D}_{3} \in \mathscr{D}_{F}^{\circ}(\mathfrak{a}), \tilde{D}_{1} \in \mathscr{D}_{F}^{\circ}\left(\mathfrak{a}^{\prime}\right)$ and $\tilde{D}_{2} \in \mathscr{D}_{F}^{\circ}\left(\mathfrak{a}^{\prime \prime}\right)$ such that

$$
\mathfrak{a}_{D_{3}}^{1}=\mathfrak{a}_{\tilde{D}_{1}}^{\prime}=\mathfrak{a}_{\tilde{D}_{2}}^{\prime \prime}=\mathfrak{g}
$$


so that we have the commutative diagram

$(* * * *)$

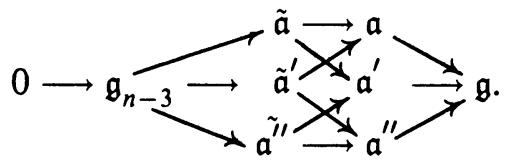

The structure $(\mathfrak{g}, \rho)$ is given by the truncated structure $\left(\mathfrak{g}_{n-3}, \rho_{0}, \pi_{I, I}^{F}\left(\mathfrak{g}_{n-3}\right)\right)$, with

$$
\pi_{I, I}^{F}\left(g_{n-3}\right)=\left(A_{\alpha}, U_{\alpha \beta} \mid(\alpha, \beta) \in I^{2}\right\} .
$$

The data $K_{i, I}^{F}\left(\mathfrak{g}_{n-3}\right)$ and $\left\{\rho_{0}, \pi_{I, I}^{F}\left(\mathfrak{g}_{n-3}\right)\right)$ are connected as in Definition 2.2. Now we consider an analogous construction in $\mathfrak{g}^{\omega}$ by taking $\pi^{-1}(* * * *)$, so that we get the following diagram:
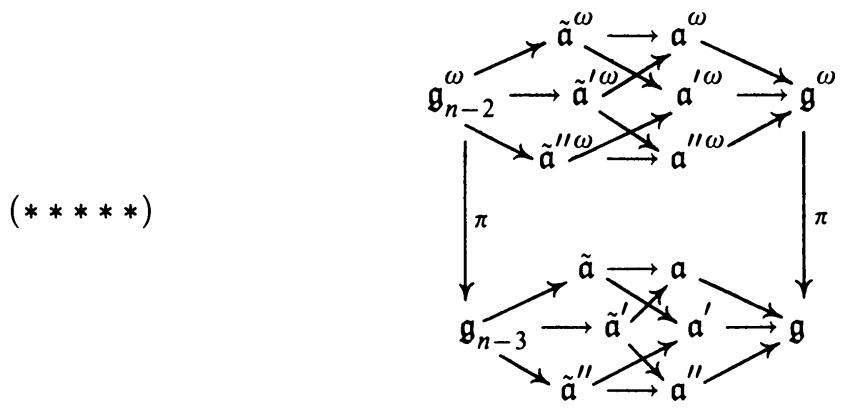

By the virtue of hypothesis (i), the structures $\rho_{\mathfrak{a}}, \rho_{a^{\prime}}$, and $\rho_{a^{\prime \prime}}$ have the $[\omega]$ lifting property. Let $\left(\mathfrak{a}^{\omega}, \rho_{\mathfrak{a}}^{\omega}\right),\left(\mathfrak{a}^{\prime \omega}, \rho_{\mathfrak{a}^{\prime}}^{\omega}\right)$ and $\left(\mathfrak{a}^{\prime \prime \omega}, \rho_{\mathfrak{a}^{\prime \prime}}^{\omega}\right)$ be the corresponding lifted structures.

We consider $\left(\mathfrak{a}^{\omega}, \rho_{\mathfrak{a}}^{\omega},\left(\mathfrak{a}^{\prime \omega}, \rho_{\mathfrak{a}^{\prime}}^{\omega}\right)\right.$ and $\left(\mathfrak{a}^{\prime \prime}, \rho_{\mathfrak{a}^{\prime \prime}}^{\omega}\right)$ as truncated structures $\left(\mathfrak{g}_{n-2}^{\omega}, \tilde{\rho}, \pi_{J, J}^{F}\right),\left(\mathfrak{g}_{n-2}^{\omega}, \tilde{\rho}^{\prime}, \pi_{J, J}^{\prime F}\right)$ and $\left(\mathfrak{g}_{n-2}^{\omega}, \tilde{\rho}^{\prime \prime}, \pi_{J, J}^{\prime \prime F}\right)$ respectively. Let us set

$$
\begin{aligned}
\pi_{J, J}^{F} & =\left\{\tilde{A}_{1}, \tilde{A}_{2}, \tilde{U}_{12}, \tilde{U}_{21}, \tilde{U}_{11}, \tilde{U}_{22}\right\}, \\
\pi_{J, J}^{\prime F} & =\left\{\tilde{A}_{1}^{\prime}, \tilde{A}_{3}^{\prime}, \tilde{U}_{13}, \tilde{U}_{1}^{\prime}, \tilde{U}_{33}^{\prime}\right\}, \\
\pi_{J, J}^{\prime \prime F} & =\left\{\tilde{A}_{2}^{\prime \prime}, \tilde{A}_{3}^{\prime \prime}, \tilde{U}_{23}, \tilde{U}_{32}, \widetilde{U}_{22}^{\prime \prime}, \tilde{U}_{33}^{\prime \prime}\right\} .
\end{aligned}
$$

Let $K_{I, I}^{F}\left(\mathfrak{g}_{n-2}^{\omega}\right)=\left\{\widetilde{D}_{1}, \widetilde{D}_{2}, \widetilde{D}_{3}, \widetilde{\xi}_{12}, \widetilde{\xi}_{13}, \widetilde{\xi}_{23}\right\}$ be the data such that $\mathfrak{g}^{\omega}=$ $\left(\mathfrak{g}_{n-2}^{\omega}, K_{I, I}^{F}\left(\mathfrak{g}_{n-2}^{\omega}\right)\right)$. Then we have

$$
\begin{aligned}
\mathfrak{a}^{\omega} & =\left(\mathfrak{g}_{n-2}^{\omega},\left\{\widetilde{D}_{1}, \widetilde{D}_{2}, \widetilde{\xi}_{12}\right\}\right)=\left(\mathfrak{g}_{n-2}^{\omega}, K_{J, J}^{F}\right), \\
\mathfrak{a}^{\prime \omega} & =\left(\mathfrak{g}_{n-2}^{\omega},\left\{\widetilde{D}_{1}, \widetilde{D}_{3}, \widetilde{\xi}_{13}\right\}\right)=\left(\mathfrak{g}_{n-2}^{\omega}, K_{J, J}^{\prime F}\right), \\
\mathfrak{a}^{\prime \prime \omega} & =\left(\mathfrak{g}_{n-2}^{\omega},\left\{\widetilde{D}_{2}, \widetilde{D}_{3}, \boldsymbol{\xi}_{23}\right\}=\left(\mathfrak{g}_{n-2}^{\omega}, K_{J, J}^{\prime \prime}\right) .\right.
\end{aligned}
$$

We know that $\left(\mathfrak{g}_{n-2}^{\omega}, \tilde{\rho}, \pi_{J, J}^{F}\right)$ and $K_{J, J}^{F}$ are connected by the relations $\left(k_{\alpha}\right)$, $\left(k_{\alpha, \beta}\right),\left(k_{\alpha, \beta, \gamma}\right)$ and $\left(k_{J, \gamma_{0}}\right)_{\alpha, \beta, \gamma}$. The same assertions hold for the couples 
$\left\{\left(\mathfrak{g}_{n-2}^{\omega}, \tilde{\rho}^{\prime}, \pi_{J, J}^{\prime F}\right), K_{J, J}^{\prime F}\right\}$ and $\left\{\left(\mathfrak{g}_{n-2}^{\omega}, \tilde{\rho}^{\prime \prime}, \pi_{J, J}^{\prime \prime F}\right), K_{J, J}^{\prime \prime F}\right\}$. By the virtue of hypothesis (ii) we have

$$
\rho_{\mathfrak{a}||^{\omega} \omega}^{\omega}=\rho_{\mathfrak{a}^{\prime} \mid \tilde{a}^{\prime}}^{\omega}=\rho_{\tilde{a}}^{\omega}, \quad \rho_{\mathfrak{a} \mid \tilde{a}^{\prime} \omega}^{\omega}=\rho_{\mathfrak{a}^{\prime \prime} \mid \tilde{a}^{\prime} \omega}^{\omega}=\rho_{\tilde{a}}^{\omega}, \quad \rho_{\mathfrak{a}^{\prime} \mid \tilde{a}^{\prime \prime} \omega}^{\omega}=\rho_{\mathfrak{a}^{\prime} \mid \tilde{a}^{\prime \prime} \omega}^{\omega}=\rho_{\tilde{a}^{\prime \prime}}^{\omega} .
$$

On the other hand, the hypothesis (ii) tells us that

$$
\rho_{\hat{a} \mid g_{n-2}^{\omega}}^{\omega}=\rho_{\tilde{\mathfrak{a}}^{\prime} \mid g_{n-2}^{\omega}}^{\omega}=\rho_{\tilde{\mathfrak{a}}^{\prime \prime} \mid \mathfrak{g}_{n-2}^{\omega}}^{\omega}=\rho_{0}^{\omega},
$$

so that we obtain

$$
\tilde{\rho}=\tilde{\rho}^{\prime}=\tilde{\rho}^{\prime \prime}=\rho_{0}^{\omega} .
$$

These equalities imply the following

$$
\begin{array}{rlrl}
\tilde{A}_{1} & =\tilde{A}_{1}^{\prime}, & & \tilde{U}_{11}=\widetilde{U}_{11}^{\prime} ; \\
\tilde{A}_{3}{ }^{\prime}=\tilde{A}_{3}^{\prime \prime}=\tilde{A}_{3}, & \tilde{U}_{33}^{\prime}=\widetilde{U}_{33}^{\prime \prime}=\widetilde{U}_{33} ; \\
\tilde{A}_{2}=\tilde{A}_{2}^{\prime \prime}, & \widetilde{U}_{22}=\tilde{U}_{22}^{\prime \prime} .
\end{array}
$$

Thus, by setting $\pi_{I, I}^{F}\left(\mathfrak{g}_{n-2}^{\omega}\right)=\left\{\tilde{A}_{\alpha}, \widetilde{U}_{\alpha, \beta} \mid(\alpha, \beta) \in\{1,2,3\}^{2}\right\}$ we see that if the data $\left(\mathfrak{g}_{n-2}^{\omega}, \rho_{0}^{\omega}, \pi_{I, I}^{F}\left(\mathfrak{g}_{n-2}^{\omega}\right)\right)$ and $K_{I, I}^{F}\left(\mathfrak{g}_{n-2}^{\omega}\right)$ are connected by the conditions $\left(k_{\alpha}\right),\left(k_{\alpha, \beta}\right),\left(k_{\alpha, \beta, \gamma}\right)$ and $\left(k_{I, \gamma_{0}}\right)_{\alpha, \beta, \gamma} ;$ this is equivalent to saying that $\rho$ has the $[\omega]$-lifting property.

Since $a_{3}$ is commutative the $v_{\alpha \beta}^{\gamma^{\prime} s}$ are zero; so that it is easy to see that the conditions $\left(k_{\alpha}\right),\left(k_{\alpha \beta}\right)$ and $\left(k_{I, \gamma_{0}}\right)$ hold. Let us prove that $\left(k_{\alpha \beta \gamma}\right)$ holds also. We consider the 2-dimensional ideal of $a_{3}$ spanned by $e_{\alpha}+e_{\beta}$ and $e_{\alpha}+e_{\gamma}$. Then by hypothesis (i) the Lifting Theorem holds for the $n$-dimensional ideal $\mathfrak{b}=\mathfrak{g}_{n-2} \oplus R\left(e_{\alpha}+e_{\beta}\right) \oplus R\left(e_{\alpha}+e_{\gamma}\right)$. By writing that the following identity holds

$$
\begin{aligned}
\rho_{\mathrm{b}}\left(\rho_{\mathrm{b}}\left(e_{\alpha}+e_{\beta}\right)\left(e_{\alpha}+e_{\gamma}\right)\right)\left(\lambda\left(e_{\alpha}+e_{\beta}\right)+\mu\left(e_{\alpha}+e_{\gamma}\right)\right) \\
-\rho_{\mathrm{b}}\left(e_{\alpha}+e_{\beta}\right) \rho_{\mathrm{b}}\left(e_{\alpha}+e_{\gamma}\right)\left(\lambda\left(e_{\alpha}+e_{\beta}\right)+\mu\left(e_{\alpha}+e_{\gamma}\right)\right) \\
=\rho_{\mathrm{b}}\left(\rho_{\mathrm{b}}\left(e_{\alpha}+e_{\gamma}\right)\left(e_{\alpha}+e_{\beta}\right)\right)\left(\lambda\left(e_{\alpha}+e_{\beta}\right)+\mu\left(e_{\alpha}+e_{\gamma}\right)\right) \\
-\rho_{\mathrm{b}}\left(e_{\alpha}+e_{\gamma}\right) \rho_{\mathrm{b}}\left(e_{\alpha}+e_{\beta}\right)\left(\lambda\left(e_{\alpha}+e_{\beta}\right)+\mu\left(e_{\alpha}+e_{\lambda}\right)\right)
\end{aligned}
$$

for all the $(\lambda, \mu) \in R^{2}$, one obtains the following equalities

$$
\begin{aligned}
\left(A_{\alpha}-D_{\alpha}\right) \xi_{\gamma \beta}-A_{\gamma} U_{\beta \alpha}+A_{\beta} U_{\gamma \alpha} & =\left(A_{\beta}-D_{\beta}\right) \xi_{\alpha \gamma}-A_{\alpha} U_{\gamma \beta}+A_{\gamma} U_{\alpha \beta} \\
& =\left(A_{\gamma}-D_{\gamma}\right) \xi_{\beta \alpha}-A_{\beta} U_{\alpha \gamma}+A_{\alpha} U_{\beta \gamma} .
\end{aligned}
$$

Because of the condition, $\xi_{\alpha \beta}=U_{\alpha \beta}-U_{\beta \alpha}$ we see that

$$
3\left[\left(A_{\alpha}-D_{\alpha}\right) \xi_{\gamma \beta}-A_{\gamma} U_{\beta \alpha}+A_{\beta} U_{\gamma \alpha}\right]=D_{\alpha} \xi_{\beta \gamma}-D_{\beta} \xi_{\alpha \gamma}+D_{\gamma} \xi_{\alpha \beta} .
$$

By virtue of $\left(C_{\alpha \beta \gamma}\right)$ we see that

$$
\left(A_{\alpha}-D_{\alpha}\right) \xi_{\gamma \beta}-A_{\gamma} U_{\beta \alpha}+A_{\beta} U_{\gamma \alpha}=0
$$

so that $\left(k_{\alpha \beta, \gamma}\right)$ holds. 
Case 2. $\mathfrak{a}_{3}$ is the Heisenberg algebra $\mathfrak{h}_{3}$. In this case the quotient structure $\left(\mathfrak{h}_{3}, \bar{\rho}\right)$ is given in the basis $\left(\bar{e}_{1}, \bar{e}_{2}, \bar{e}_{3}\right)$ by the matrix

$$
\bar{\rho}\left(x_{1}, x_{2}, x_{3}\right)=\left[\begin{array}{ccc}
0 & a x_{3} & (a+\theta) x_{2} \\
0 & 0 & \\
0 & 0 & 0
\end{array}\right]
$$

where the scalar $\theta \in \mathbf{R}$ is defined through

$$
\left[\bar{e}_{2}, \bar{e}_{3}\right]=\theta \bar{e}_{1} .
$$

By changing $\left\|\bar{e}_{1}\right\|$ we can take $\theta=1$. Then the Lie algebra $\mathfrak{g}$ is given by the truncated Lie algebra $\left(\mathfrak{g}_{n-3}, K_{I, I}^{F}\left(\mathfrak{g}_{n-3}\right)\right)$. We have $K_{I, I}^{F}\left(\mathfrak{g}_{n-3}\right)=\left\{D_{1}, D_{2}, D_{3}\right.$, $\left.\xi_{12}, \xi_{23}, \varepsilon_{23}^{1}=1\right\}$. We set $v_{32}^{1}=a$, so that the structure $(\mathfrak{g}, \rho)$ is given by the truncated structure

$$
\left(\mathfrak{g}_{n-3}, \rho_{0}, \pi_{I, I}^{F}\left(\mathfrak{g}_{n-3}\right)\right)
$$

and

$$
\pi_{I, I}^{F}\left(\mathfrak{g}_{n-3}\right)=\left\{A_{\alpha}, U_{\alpha \beta}, v_{\alpha \beta}^{\gamma} \mid(\alpha, \beta, \gamma) \in\{1,2,3\}^{3}\right\} .
$$

We consider the following ideals of $\mathfrak{h}_{3}$ :

$$
\mathfrak{a}_{2}=\operatorname{span}\left(e_{1}, e_{2}\right), \quad \mathfrak{a}_{2}^{\prime}=\operatorname{span}\left(e_{1}, e_{3}\right) .
$$

Then the ideals $\mathfrak{a}=\tilde{\pi}^{-1}\left(\mathfrak{a}_{2}\right)$ and $\mathfrak{a}^{\prime}=\tilde{\pi}^{-1}\left(\mathfrak{a}_{2}^{\prime}\right)$ are spanned by $\left(e_{1}, \ldots, e_{n-3}\right.$, $\left.e_{n-2}, e_{n-1}\right)$ and $\left(e_{1}, \ldots, e_{n-3}, e_{n-2}, e_{n}\right)$ respectively. We have the following diagram:

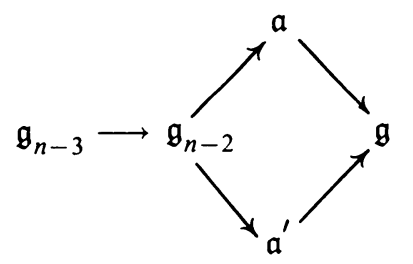

If we take the pull back in $\mathfrak{g}^{\omega}$ of this diagram, we get

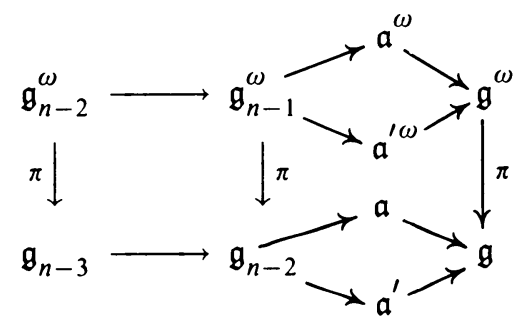

By virtue of hypothesis (i), $\rho_{a}$ and $\rho_{a^{\prime}}$ have the [ $\left.\omega\right]$-lifting property. Let $\left(\mathfrak{a}^{\omega}, \rho_{\mathfrak{a}}^{\omega}\right)$ and $\left(\mathfrak{a}^{\prime \omega}, \rho_{\mathfrak{a}^{\prime}}^{\omega}\right)$ be the corresponding lifted structure. 
We consider $\mathfrak{g}^{\omega}$ as the truncated Lie algebra $\left(\mathfrak{g}_{n-2}^{\omega}, K_{I, I}^{F}\right)$ where

$$
K_{I, I}^{F}=\left\{\widetilde{D}_{\alpha}, \tilde{\xi}_{\alpha \beta}, \varepsilon_{23}^{1}=1 \mid(\alpha, \beta) \in\{1,2,3\}^{2}\right\} .
$$

Therefore we identify $\mathfrak{a}^{\omega}$ (resp. $\left.\mathfrak{a}^{\prime \omega}\right)$ with the truncated Lie algebra $\left(\mathfrak{g}_{n-2}^{\omega}\right.$, $\left.K_{J, J}^{F}\right)\left(\operatorname{resp} .\left(\mathfrak{g}_{n-2}^{\omega}, K_{J, J}^{\prime F}\right)\right)$ where

$$
K_{J, J}^{F}=\left\{\widetilde{D}_{1}, \widetilde{D}_{2}, \widetilde{\xi}_{12}\right\}, \quad\left(\text { resp. } K_{J, J}^{\prime F}=\left\{\widetilde{D}_{1}, \widetilde{D}_{3}, \widetilde{\xi}_{13}\right\}\right)
$$

According to our previous notations we set

$$
\left(\mathfrak{a}^{\omega}, \rho_{\mathfrak{a}}^{\omega}\right)=\left(\mathfrak{g}_{n-2}^{\omega}, \tilde{\rho}, \pi_{J, J}^{F}\right), \quad\left(\mathfrak{a}^{\prime \omega}, \rho_{\mathfrak{a}^{\prime}}^{\omega}\right)=\left(\mathfrak{g}_{n-2}^{\omega}, \tilde{\rho}^{\prime}, \pi_{J, J}^{\prime F}\right)
$$

with

$$
\begin{aligned}
\pi_{J, J}^{F} & =\left\{\tilde{A}_{1}, \tilde{A}_{2}, \tilde{U}_{12}, \tilde{U}_{21}, U_{11} \tilde{U}_{22}\right\} \\
\pi_{J, J}^{\prime F} & =\left\{\tilde{A}_{1}^{\prime}, \tilde{A}_{3}, \tilde{U}_{13}, \tilde{U}_{31}, \widetilde{U}_{11}^{\prime}, \widetilde{U}_{33}\right\}
\end{aligned}
$$

By virtue of hypothesis (ii) we have

$$
\rho_{\mathfrak{a} \mid \mathfrak{g}_{n-1}^{\omega}}^{\omega}=\rho_{\mathfrak{a}^{\prime} \mid \mathfrak{g}_{n-1}^{\omega}}^{\omega}
$$

so that we have $\tilde{\rho}=\tilde{\rho}^{\prime}=\rho_{0}^{\omega}, \tilde{A}_{1}=\tilde{A}_{1}$ and $\tilde{U}_{11}=\tilde{U}_{11}{ }^{\prime}$. Therefore the data $\pi_{J, J}^{F}$ and $\pi_{J, J}^{F}$ satisfy the following system:

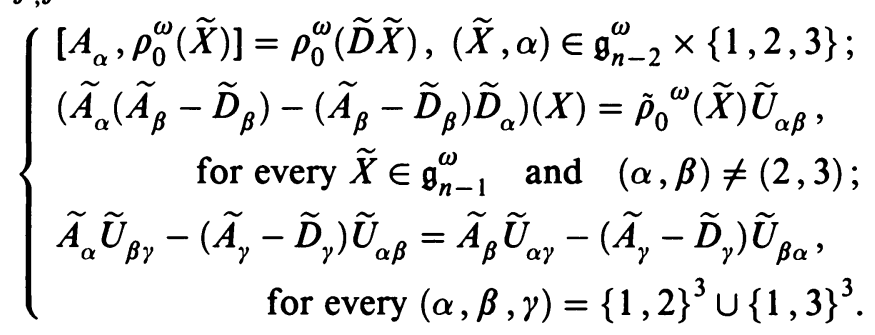

One easily sees that the system $(\sigma)$ depends only on $\pi\left(\tilde{U}_{\alpha \beta}\right)$. Therefore we set

$$
\tilde{U}_{\alpha \beta}=\left(u_{\alpha \beta}, U_{\alpha \beta}\right) \in \mathfrak{g}_{n-2}^{\omega}=\mathbf{R} \times \mathfrak{g}_{n-3} .
$$

Let us examine the matrix associated to each $\tilde{A}_{\alpha}$ in the basis $\left((1,0), e_{1}, \ldots\right.$, $\left.e_{n-3}\right)$ of $\mathfrak{g}_{n-2}^{\omega}$. We set

$$
\tilde{A}_{\alpha}=\left[\begin{array}{ll}
0 & a_{\alpha} \\
0 & A_{\alpha}
\end{array}\right], \quad \tilde{D}_{\alpha}=\left[\begin{array}{cc}
0 & \theta_{\alpha} \\
0 & D_{\alpha}
\end{array}\right],
$$

where $a_{\alpha} \in \mathfrak{g}_{n-3}^{*}$. It is clear that the $A_{\alpha}$ are those which give $(\mathfrak{g}, \rho)=$ $\left(\mathfrak{g}_{n-3}, \rho_{0}, \pi_{I, I}^{F}\right)$. Let $\varphi \in C^{1}\left(\mathfrak{g}_{n-3}, \mathfrak{g}_{n-3}^{*}\right)$ be such that $\rho_{0}^{\omega}$ is given by

$$
\rho_{0}^{\omega}(x, X)(y, Y)=\left(\varphi(X, Y), \rho_{0}(X) Y\right) \text {. }
$$

By considering $\mathfrak{g}_{n-1}^{\omega}$ as the Lie algebra $\left(\mathfrak{g}_{n-2}^{\omega}\right) \widetilde{D}_{1}, \rho_{0}^{\omega}$ has the $\widetilde{D}_{1}$-extension property. In other words, $a_{\alpha}, \theta_{\alpha}$ and $\varphi$ must be connected by the following conditions:

$$
\left\{\begin{array}{l}
\varphi\left(D_{\alpha} X, Y\right)+\varphi\left(X, A_{\alpha} Y\right)-a_{\alpha}\left(\rho_{0}(X) Y\right)=0, \\
a_{\alpha}\left(\left(A_{\alpha}-D_{\alpha}\right) X\right)-\left(a_{\alpha}-\theta_{\alpha}\right)\left(D_{\alpha} X\right)-\varphi\left(X, U_{\alpha \alpha}\right)=0 .
\end{array}\right.
$$


These relations are direct consequence of $\left(k_{\alpha}\right)$ and $\left(k_{\alpha \beta}\right)$, respectively. We saw that the quotient structure $\left(\mathfrak{h}_{3}, \bar{\rho}\right)$ has the matrix

$$
\bar{\rho}\left(x_{1}, x_{2}, x_{3}\right)=\left[\begin{array}{ccc}
0 & a x_{3} & (1+a) x_{2} \\
0 & 0 & 0 \\
0 & 0 & 0
\end{array}\right],
$$

where $a$ is a fixed real number. Let us set $\left(\bar{\varepsilon}_{1}, \bar{\varepsilon}_{2}, \bar{\varepsilon}_{3}\right)=\left(\lambda e_{1}, u \bar{e}_{2}+v \bar{e}_{3}, \mu \bar{e}_{2}+\right.$ $\left.\nu \vec{e}_{3}\right)$ with $\lambda=u \nu-v \mu \neq 0$. Then we have

$$
\left[\bar{\varepsilon}_{2}, \bar{\varepsilon}_{3}\right]=(u \nu-v \mu) \bar{e}_{1}=\bar{\varepsilon}_{1},
$$

and

$$
\begin{aligned}
\bar{\rho}\left(\bar{\varepsilon}_{2}\right) \bar{\varepsilon}_{3} & =\bar{\rho}\left(u \bar{e}_{2}+v \bar{e}_{3}\right)\left(\mu \bar{e}_{2}+\nu \bar{e}_{3}\right)=\nu \bar{\rho}\left(\bar{e}_{2}\right) \bar{e}_{3}+v \mu \bar{\rho}\left(\bar{e}_{3}\right) \bar{e}_{2} \\
& =(a+1) u \nu \bar{e}_{1}+v \mu a \bar{e}_{1}=[(a+1) u \nu+v \mu a] \bar{e}_{1} .
\end{aligned}
$$

Let us look for $(u, v, \mu, \nu)$ such that

$$
(a+1) u \nu+v \mu a=\frac{1}{2}(u \nu-v \mu) .
$$

That will give

$$
2(a+1) u \nu+2 v \mu a=u \nu-v \mu
$$

so that we get

$$
(2 a+1)(u \nu+v \mu)=0 .
$$

Therefore one sees that if $a \neq-\frac{1}{2}$ one can choose some $(u, v, \nu, \mu)$ such that $u \nu=-v \mu \neq 0$. Thus, we can always choose some basis $\left(e_{1}, \ldots, e_{n}\right)$ in $\mathfrak{g}$ such that $\left[\bar{e}_{2} \bar{e}_{3}\right]=\bar{e}_{1}$ and the matrix of $\bar{\rho}$ in $\left(\bar{e}_{1}, \bar{e}_{2}, \bar{e}_{3}\right)=\left(\bar{e}_{n-2}, \bar{e}_{n-1}, \bar{e}_{n}\right)$ is

$$
\bar{\rho}\left(x_{1}, x_{2}, x_{3}\right)=\left[\begin{array}{ccc}
0 & -\frac{x_{3}}{2} & \frac{x_{2}}{2} \\
0 & 0 & 0 \\
0 & 0 & 0
\end{array}\right] .
$$

We fix such basis; we need the following lemma:

Lemma 4.1. The data $\left(a_{\alpha}, \theta_{\alpha}, A_{\alpha}, D_{\alpha}, U_{\alpha \beta} \mid(\alpha, \beta) \in\{2,3\}^{2}\right)$ and $\varphi$ are connected by the formula $a_{2}\left(\left(A_{3}-D_{3}\right) X\right)-\left(a_{3}-\theta_{3}\right)\left(D_{2} X\right)-\varphi\left(X, U_{23}\right)=$ $-a_{3}\left(A_{2}-D_{2}\right) X+\left(a_{2}-\theta_{2}\right)\left(D_{3} X\right)+\varphi\left(X, U_{32}\right), X \in \mathfrak{g}_{n-3}$.

Proof. We consider the ideal $\overline{\mathfrak{a}}_{\lambda, \mu} \subset \mathfrak{h}_{3}$ spanned by $\left(\bar{e}_{1}, \lambda \bar{e}_{2}+\mu \bar{e}_{3}\right)$. Let $a_{\lambda \mu}=$ $\pi^{-1}\left(\overline{\mathfrak{a}}_{\lambda \mu}\right) \subset \mathfrak{g}$. The ideal $\mathfrak{a}_{\lambda \mu}^{\omega}$ can be identified with $\left(\mathfrak{g}_{n-2}^{\omega}\right)_{\widetilde{D}_{\lambda \mu}}$ where $\widetilde{D}_{\lambda \mu}=$ $\lambda \widetilde{D}_{2}+\mu \widetilde{D}_{3}$. By virtue of hypothesis (ii) of Theorem 4.1, $\rho_{0}^{\omega}$ has the $\widetilde{D}_{\lambda \mu}$ extension property, so that if we set $A_{\lambda \mu}=\lambda \widetilde{A}_{2}+\mu \widetilde{A}_{3}$ then there is $\widetilde{U}_{\lambda \mu}$ such that $\lambda \widetilde{D}_{2}+\mu \widetilde{D}_{3}$ satisfying

$$
\left[\tilde{A}_{\lambda \mu}, \rho_{0}^{\omega}(\widetilde{X})\right]=\rho_{0}^{\omega}\left(\widetilde{D}_{\lambda \mu} \widetilde{X}\right)
$$


and $\left(\widetilde{A}_{\lambda \mu}^{2}+\widetilde{D}_{\lambda \mu}^{2}-2 \widetilde{A}_{\lambda \mu} \widetilde{D}_{\lambda \mu}\right) \widetilde{X}=\rho_{0}^{\omega}(\widetilde{X}) \widetilde{U}_{\lambda \mu}$. The latter condition gives

$$
\begin{aligned}
\left(\left[\lambda \widetilde{A}_{2}\right.\right. & \left.\left.+\mu \widetilde{A}_{2}\right]^{2}+\left(\lambda \widetilde{D}_{2}+\mu \widetilde{D}_{3}\right)^{2}-2\left(\lambda \widetilde{A}_{2}+\mu \widetilde{A}_{3}\right)\left(\lambda \widetilde{D}_{2}+\mu \widetilde{D}_{3}\right)\right)(\widetilde{X})=\rho_{0}^{\omega}(\widetilde{X}) \widetilde{U}_{\lambda \mu} \\
= & \rho_{0}^{\omega}(\widetilde{X})\left(\lambda^{2} \widetilde{U}_{22}+\mu^{2} \widetilde{U}_{33}\right) \\
& +\lambda \mu\left(\tilde{A}_{2}\left(\tilde{A}_{3}-\widetilde{D}_{3}\right)-\left(\widetilde{A}_{3}-\widetilde{D}_{3}\right) \widetilde{D}_{3}+\widetilde{A}_{3}\left(\widetilde{A}_{2}-\widetilde{D}_{2}\right)-\left(\widetilde{A_{2}}-\widetilde{D}_{2}\right) \widetilde{D}_{3}\right) \cdot \tilde{X} .
\end{aligned}
$$

But, by projecting this condition in $\mathfrak{g}_{n-3}$, we can choose $\tilde{U}_{\lambda \mu}=\left(u_{\lambda \mu} U_{\lambda \mu}\right)$ with $U_{\lambda \mu}=\lambda_{22}^{2} U_{22}+\mu^{2} U_{33}+\lambda \mu\left(U_{23}+U_{32}\right)$, so that by taking the $(\mathbf{R} \times 0)$-component we see that

$$
\begin{aligned}
\varphi\left(X, U_{\lambda \mu}\right)= & \phi\left(X, \lambda^{2} U_{22}+\mu^{2} U_{33}+\lambda \mu\left(U_{23}+U_{32}\right)\right) \\
=\phi\left(X, \lambda^{2} U_{22}+\mu^{2} U_{33}\right)+\lambda \mu\left(a_{2}\left(\left(A_{3}-D_{3}\right) \cdot X\right)-\left(a_{3}-\theta_{3}\right)\left(D_{2} X\right)\right. & \left.\quad+a_{3}\left(\left(A_{2}-D_{2}\right) \cdot X\right)-\left(a_{2}-\theta_{2}\right)\left(D_{3} X\right)\right) .
\end{aligned}
$$

Finally we obtain the following equality:

$$
\begin{aligned}
& \varphi\left(X, U_{23}+U_{32}\right)=a_{2}\left(\left(A_{3}-D_{3}\right) \cdot X\right)-\left(a_{3}-\theta_{3}\right)\left(D_{2} X\right) \\
& +a_{3}\left(\left(A_{2}-D_{2}\right) \cdot X\right)-\left(a_{2}-\theta_{2}\right)\left(D_{3} X\right) .
\end{aligned}
$$

for every $X \in \mathfrak{g}_{n-3}$. Lemma 4.1 is proved

Now we define the linear form $a_{1}^{\prime} \in \mathfrak{g}_{n-3}^{*}$ by setting

$$
\begin{aligned}
\frac{1}{2}\left(a_{1}^{\prime}-\theta_{1}\right)(X) & =a_{2}\left(\left(A_{3}-D_{3}\right) \cdot X\right)-\left(a_{3}-\theta_{3}\right)\left(D_{2} X\right)-\phi\left(X, U_{23}\right) \\
& =-a_{3}\left(\left(A_{2}-D_{2}\right) \cdot X\right)+\left(a_{2}-\theta_{2}\right)\left(D_{3} X\right)+\phi\left(X, U_{32}\right) .
\end{aligned}
$$

This formula gives

$$
\begin{aligned}
\left(a_{1}^{\prime}-\theta_{1}\right)(X)= & \left.a_{2}\left(A_{3}-D_{3}\right) \cdot X\right)-a_{3}\left(\left(A_{2}-D_{2}\right) X\right)+\left(a_{2}-\theta_{2}\right)\left(D_{3} X\right) \\
& -\left(a_{3}-\theta_{3}\right)\left(D_{2} X\right)+\varphi\left(X, U_{23}-\xi_{23}\right)-\varphi\left(X, U_{23}\right) \\
= & a_{2}\left(A_{3} X\right)-a_{3}\left(A_{2} X\right)+\theta_{3}\left(D_{2} X\right)-\theta_{2}\left(D_{3} X\right)-\varphi\left(X, \xi_{23}\right) .
\end{aligned}
$$

We know that $\left[\widetilde{D}_{2}, \widetilde{D}_{3}\right]=\widetilde{D}_{1}+\operatorname{ad} \xi_{23}$, which implies that

$$
\theta_{2}\left(D_{3} X\right)-\theta_{3}\left(D_{2} X\right)=\theta_{1}(X)+\omega\left(\xi_{23}, X\right) \text {. }
$$

We have also $\varphi\left(\xi_{23}, X\right)-\varphi\left(X, \xi_{23}\right)=\omega\left(\xi_{23}, X\right)$, so that $a_{1}^{\prime}$ is given by

$$
a_{1}^{\prime}(X)=a_{2}\left(A_{3} X\right)-a_{3}\left(A_{2} X\right)-\varphi\left(\xi_{23}, X\right) \text {. }
$$

We need a second lemma:

Lemma 4.2. The 1-cochain $X \rightarrow \varphi\left(D_{1} X,-\right)+\varphi\left(-, A_{1} X\right)$ is the coboundary of the 0-cochain $a_{1}^{\prime}$.

Proof. We have to prove that $\varphi\left(D_{1} X, Y\right)+\varphi(X, A, Y)=a_{1}^{\prime}\left(\rho_{0}(X) Y\right)$. Indeed we have

$$
\begin{aligned}
a_{1}^{\prime}(\rho(X) Y)= & a_{2}\left(A_{3} \cdot \rho_{0}(X) Y\right)-a_{3}\left(A_{2} \rho_{0}(X) Y\right)-\varphi\left(\xi_{23}, \rho_{0}(X) Y\right) \\
= & a_{2}\left(\rho_{0}(X) A_{3} Y+\rho_{0}\left(D_{3} X\right) Y\right)-a_{3}\left(\rho_{0}(X) A_{2} Y+\rho_{0}\left(D_{2} X\right) Y\right) \\
& -\varphi\left(\left[\xi_{23}, X\right], Y\right)-\varphi\left(X, \rho_{0}\left(\xi_{23}\right) Y\right) .
\end{aligned}
$$


We know that $\left(A_{\alpha}, D_{\alpha}, a_{\alpha}\right), \alpha \in\{2,3\}$ are connected as in (iv*). Then we have

$$
\begin{aligned}
a_{1}^{\prime}\left(\rho_{0}(X) Y\right)= & \varphi\left(D_{2} X, A_{3} Y\right)+\varphi\left(X, A_{2} A_{3} Y\right)+\varphi\left(D_{2} D_{3} X, Y\right)+\varphi\left(D_{3} X, A_{2} Y\right) \\
& -\varphi\left(D_{3} X, A_{2} Y\right)-\varphi\left(X, A_{3} A_{2} Y\right)-\varphi\left(D_{3} D_{2} X, Y\right) \\
& -\varphi\left(D_{2} X, A_{3} Y\right)-\varphi\left(\left[\xi_{23}, X\right], Y\right)-\varphi\left(X, \rho_{0}\left(\xi_{23}\right) Y\right) \\
= & \varphi\left(X,\left[A_{2}, A_{3}\right] Y\right)+\varphi\left(\left[D_{2}, D_{3}\right] X, Y\right) \\
& -\varphi\left(\rho_{0}\left[\xi_{23}, X\right] Y\right)-\varphi\left(X, \rho_{0}\left(\xi_{23}\right) Y\right) \\
= & \varphi\left(\left(\left[D_{2}, D_{3}\right]-\operatorname{ad} \xi_{23}\right) X, Y\right)+\varphi\left(X,\left(\left[A_{2}, A_{3}\right]-\rho_{0}\left(\xi_{23}\right)\right) Y\right) .
\end{aligned}
$$

We know also that $\left[D_{2}, D_{3}\right]-\operatorname{ad} \xi_{23}=D_{1}$ and $\left[A_{2}, A_{3}\right]-\rho_{0}\left(\xi_{23}\right)=A_{1}$. It follows that $a_{1}^{\prime}(\rho(X) Y)=\varphi\left(D_{1} X, Y\right)+\varphi\left(X, A_{1} Y\right)$. Lemma 4.2 is proved.

We define the linear operator $\tilde{A}_{1}^{\prime}: \mathfrak{g}_{n-2}^{\omega} \rightarrow \mathfrak{g}_{n-2}^{\omega}$ be setting

$$
\tilde{A}_{1}=\left[\begin{array}{ll}
0 & a_{1}^{\prime} \\
0 & A_{1}
\end{array}\right] \text {. }
$$

The Lemma 4.2 implies that for every $\widetilde{X} \in \mathfrak{g}_{n-3}^{\omega}$,

$$
\left[\widetilde{A}_{1}, \rho_{0}^{\omega}(\widetilde{X})\right]=\rho_{0}^{\omega}\left(\widetilde{D}_{1} \widetilde{X}\right) ;
$$

so that for every pair $(X, Y) \in \mathfrak{g}_{n-3}^{2}$ we have

$$
a_{1}\left(\rho_{0}(X) Y\right)=a_{1}^{\prime}\left(\rho_{0}(X) Y\right) .
$$

Let us set $\theta_{0}(X)=a_{1}(X)-a_{1}^{\prime}(X)$. Let $s=\operatorname{dim}\left[\mathfrak{g}_{n-3}, \mathfrak{g}_{n-3}\right]$. Then by the condition (iii) of Theorem $4.1\left(e_{1}, \ldots, e_{s}\right) \operatorname{span}\left[\mathfrak{g}_{n-3}, \mathfrak{g}_{n-3}\right]$, and since $\rho_{0}$ is normal we get $\rho_{0}\left(\mathfrak{g}_{n-3}\right) \mathfrak{g}_{n-3}=\left[\mathfrak{g}_{n-3}, \mathfrak{g}_{n-3}\right]$, so that every 1-form $\eta=\mathfrak{g}_{n-3}^{*}$ with $\eta\left(\rho_{0}(X) Y\right)=0$ is a linear combination of $\eta_{s+1}, \ldots, \eta_{n-3}$ where $\left(\eta_{1}, \ldots, \eta_{n-3}\right)$ is the dual basis of $\left(e_{1}, \ldots, e_{n-3}\right)$.

The structure $(\mathfrak{g}, \rho)$ being normal, we have also $\rho(\mathfrak{g}) \mathfrak{g}=[\mathfrak{g}, \mathfrak{g}]=\mathfrak{g}_{n-2}$, so that every linear combination of $e_{s+1}, \ldots, e_{n-3}$ can be written in the form $A_{1} X_{1}+A_{2} X_{2}+A_{3} X_{3}$ where $X_{1}, X_{2}, X_{3}$ are linear combinations of $e_{s+1}, \ldots$, $e_{n-2}$. Since $A_{1}=\left[A_{2}, A_{3}\right]+\rho_{0}\left(\xi_{23}\right)$, we deduce that every linear combination of $e_{s+1}, \ldots, e_{n-3}$ can be written $A_{2} X_{2}^{\prime}+A_{2} X_{3}^{\prime}$ where $X_{2}^{\prime}, X_{3}^{\prime}$ are linear combinations of $e_{s+1}, \ldots, e_{n-3}$.

In other words we have the equality,

$$
\operatorname{span}\left(e_{s+1}, \ldots, e_{n-3}\right)=\operatorname{span}\left(A_{2} e_{x+1}, A_{3} e_{s+1}, \ldots, A_{2} e_{n-3}, A_{3} e_{n-3}\right) .
$$

Therefore let $\eta$ be an element of the dual space $\mathfrak{g}_{n-3}^{*}$ such that $\eta\left(\rho_{0}(X) Y\right) \equiv 0$ for $X \in \mathfrak{g}_{n-3}$ and $Y \in \mathfrak{g}_{n-3}$; then $\eta$ belongs to the subspace of $\mathfrak{g}_{n-3}^{*}$ spanned by the system

By virtue of the equality,

$$
\left(\eta_{s+1}, \ldots, \eta_{n-3}\right) .
$$

$$
\operatorname{span}\left(\left\{e_{s+j} / 1 \leq j \leq n-s-3\right\}\right)=\operatorname{span}\left(\left\{A_{2} e_{s+j}, A_{3} e_{s+j} / 1 \leq j \leq n-s-3\right\}\right)
$$


the linear form $\eta$ lies in the subspace spanned by the following linear forms,

$$
\eta_{s+1} \circ A_{2}, \eta_{s+1} \circ A_{3}, \ldots, \eta_{n-3} \circ A_{2}, \eta_{n-3} \circ A_{3} .
$$

In particular the linear form $\theta_{0}=a_{1}-a_{1}^{\prime}$ lies in $\operatorname{span}\left(\eta_{s+1}, \ldots, \eta_{n-3}\right)$; so that we can find $\eta^{\prime}$ and $\eta^{\prime \prime}$ in $\operatorname{span}\left(\eta_{s+1}, \ldots, \eta_{n-3}\right)$ such that $\theta_{0}=\eta^{\prime} \circ A_{2}+\eta^{\prime \prime} \circ A_{3}$. This means that for every $X \in \mathfrak{g}_{n-3}$ we have

$$
\theta_{0}(X)=\eta^{\prime}\left(\rho\left(e_{n-1}\right) X\right)+\eta^{\prime \prime}\left(\rho\left(e_{n}\right) X\right) .
$$

Let us return to $\rho_{a}^{\omega}$ and $\rho_{a^{\prime}}^{\omega}$ and $\left(\rho_{0}^{\omega}\right)_{\widetilde{D}_{1}}$ :

$$
0 \rightarrow\left(\mathfrak{g}_{n-2}^{\omega}, \rho_{0}^{\omega}\right) \rightarrow\left(\mathfrak{g}_{n-1}^{\omega}, \rho_{\mathrm{ana^{ \prime }}}^{\omega}\right)_{\left(\mathfrak{a}^{\prime \omega}, \rho_{\mathfrak{a}^{\prime}}^{\omega}\right) .}^{\left.\pi^{\left(\mathfrak{a}^{\omega}, \rho_{\mathfrak{a}}\right)}\right)}
$$

Then, $\left(\mathfrak{a}^{\omega}, \rho_{\mathfrak{a}}^{\omega}\right)\left(\right.$ resp. $\left.\left(\mathfrak{a}^{\prime \omega}, \rho_{\mathfrak{a}^{\prime}}^{\omega}\right)\right)$ is given by $\left(\mathfrak{a}, \rho_{\mathfrak{a}}, \varphi_{\mathfrak{a}}\right)\left(\operatorname{resp} .\left(\mathfrak{a}^{\prime}, \rho_{\mathfrak{a}^{\prime}}, \varphi_{\mathfrak{a}^{\prime}}\right)\right)$ such that for $(X, Y) \in \mathfrak{a} \cap \mathfrak{a}^{\prime}$ we have

$$
\varphi_{\mathfrak{a}}(X, Y)=\varphi_{\mathfrak{a}^{\prime}}(X, Y) ;
$$

we recall that $\mathfrak{a} \cap \mathfrak{a}^{\prime}=\mathfrak{g}_{n-2}$. Let us change $\varphi_{\mathfrak{a}}$ and $\varphi_{\mathfrak{a}^{\prime}}$ by putting

$$
\tilde{\varphi}_{\mathrm{a}}(X, Y)=\varphi_{\mathrm{a}}(X, Y)+\eta^{\prime}(\rho(X) Y), \quad \tilde{\varphi}_{\mathrm{a}^{\prime}}(X, Y)=\varphi_{\mathrm{a}^{\prime}}(X, Y)-\eta^{\prime \prime}(\rho(X) Y) .
$$

It is clear that $\left(\mathfrak{a}^{\omega}, \rho_{\mathfrak{a}}, \tilde{\varphi}_{\mathfrak{a}}\right)$ (resp. $\left.\left(\mathfrak{a}^{\prime \omega}, \rho_{\mathfrak{a}}, \tilde{\varphi}_{\mathfrak{a}^{\prime}}\right)\right)$ defines a lifted structure in $\mathfrak{a}^{\omega}$ (resp. $\left.\mathfrak{a}^{\prime \omega}\right)$. We have the following

$$
\tilde{\varphi}_{\mathfrak{a}}\left(D_{2} X, Y\right)+\tilde{\varphi}_{\mathfrak{a}}\left(\left(X, A_{2} Y\right)=a_{2}(\rho(X) Y)+\eta^{\prime}\left(A_{2} \rho(X) Y\right) \quad \text { if } X, Y \in \mathfrak{a}\right.
$$

and

$$
\tilde{\varphi}_{a^{\prime}}\left(D_{3} X, Y\right)+\tilde{\varphi}_{a^{\prime}}\left(X, A_{3} Y\right)=a_{3}(\rho(X) Y)-\eta^{\prime \prime}\left(A_{3} \rho(X) Y\right), \quad \text { if } X, Y \in \mathfrak{a}^{\prime} .
$$

We know that for $(X, Y) \in \mathfrak{g}_{n-3}$ we have

$$
\eta^{\prime}\left(\rho_{0}(X) Y\right)=\eta^{\prime \prime}\left(\rho_{0}(X) Y\right)=0
$$

so that $\tilde{\varphi}_{a} \mid g_{n-3}=\tilde{\varphi}_{a^{\prime} \mid g_{n-3}}=\varphi$. We set $\tilde{a}_{2}=a_{2}+\eta^{\prime} A_{2}$ and $\tilde{a}_{3}=a_{3}-\eta^{\prime \prime} A_{3}$, so that

$$
\begin{aligned}
\tilde{a}_{1}^{\prime} & =\tilde{a}_{2} A_{3}-\tilde{a}_{3} A_{2}-\varphi\left(\xi_{23},-\right) \\
& =a_{2} A_{3}-a_{3} A_{2}-\varphi\left(\xi_{23},-\right)+\eta^{\prime} A_{2}+\eta^{\prime \prime} A_{3} \\
& =a_{1}^{\prime}+\theta_{0}=a_{1} .
\end{aligned}
$$

By virtue of this result together with Lemma 4.1 the operators

$$
\tilde{A}_{1}=\left[\begin{array}{ll}
0 & a_{1} \\
0 & A_{1}
\end{array}\right], \quad \tilde{A}_{2}=\left[\begin{array}{cc}
0 & \tilde{a}_{2} \\
0 & A_{2}
\end{array}\right] \quad \text { and } \quad \tilde{A}_{3}=\left[\begin{array}{cc}
0 & a_{3} \\
0 & A_{3}
\end{array}\right]
$$

are connected by the formulas $\left(k_{23}\right)$ and $\left(k_{32}\right)$ :

$$
\begin{gathered}
\left(\tilde{A}_{2}\left(\tilde{A}_{3}-\widetilde{D}_{3}\right)-\left(\tilde{A}_{3}-\widetilde{D}_{3}\right) \widetilde{D}_{2}-\frac{1}{2}\left(\tilde{A}_{1}-\widetilde{D}_{1}\right)\right) \tilde{X}=\rho_{0}^{\omega}(\tilde{X}) \widetilde{U}_{23}, \\
\left(\tilde{A}_{3}\left(\tilde{A}_{2}-\widetilde{D}_{2}\right)-\left(\tilde{A}_{2}-\widetilde{D}_{2}\right) \widetilde{D}_{3}+\frac{1}{2}\left(\tilde{A}_{1}-\widetilde{D}_{1}\right)\right) \cdot \tilde{X}=\rho_{0}^{\omega}(\tilde{X}) \widetilde{U}_{32},
\end{gathered}
$$


where $\tilde{X}, \tilde{U} \in \mathfrak{g}_{n-3}^{\omega}, \tilde{U}_{23}=\left(u_{23}, U_{23}\right), \widetilde{U}_{23}-\widetilde{U}_{32}=\widetilde{\xi}_{23}$. On these formulas the real number $u_{23}$ can be chosen arbitrarily. Therefore by setting $\widetilde{U}_{\alpha \beta}=$ $\left(u_{\alpha \beta}, U_{\alpha \beta}\right)$ it remains to prove that we can choose $u_{\alpha \beta}$ such that $\left(\mathfrak{g}_{n-2}^{\omega}, \rho_{0}^{\omega}, \pi_{I, I}^{F}\right)$ defines a truncated Koszul-Vinberg structure in $\left(\mathfrak{g}_{n-2}^{\omega}, K_{I, I}^{F}\right)$, with

$$
\pi_{I, I}^{F}=\left\{\tilde{A}_{\alpha}, \tilde{U}_{\alpha \beta}, v_{23}^{1}=-v_{32}^{1}=\frac{1}{2}\right\}, \quad \alpha, \beta=1,2,3 .
$$

These data satisfy the conditions $\left(k_{\alpha}\right),\left(k_{\alpha \beta}\right)$ and $\left(k_{I, \gamma_{0}}\right)_{\alpha \beta, \gamma}$. It remains to prove that there are $\left(u_{\alpha \beta}\right), \alpha, \beta=1,2,3$ such that $\left(k_{\alpha \beta \gamma}\right)$ holds. Since $\left(k_{\alpha \beta \gamma}\right)$ holds for $(\alpha, \beta, \gamma) \in\{1,2\}^{3} \cup\{1,3\}^{3}$, we have to prove that one can find $\left(u_{\alpha \beta}\right)$ such that the following system holds:

$$
\begin{aligned}
& \left(k_{123}\right): \widetilde{A}_{1} \widetilde{U}_{23}-\widetilde{A}_{2} \widetilde{U}_{13}-\left(\widetilde{A}_{3}-\widetilde{D}_{3}\right) \widetilde{\xi}_{12}=-\frac{1}{2} \widetilde{U}_{11}, \\
& \left(k_{132}\right): \tilde{A}_{1} \widetilde{U}_{32}-\tilde{A}_{3} \widetilde{U}_{12}-\left(\tilde{A}_{2}-\widetilde{D}_{2}\right) \widetilde{\xi}_{13}=\frac{1}{2} \widetilde{U}_{11} \text {, } \\
& \left(k_{231}\right): \tilde{A}_{2} \widetilde{U}_{31}-\tilde{A}_{3} \widetilde{U}_{21}-\left(\tilde{A}_{1}-\widetilde{D}_{1}\right) \xi_{23}=\widetilde{U}_{11} \text {, } \\
& \left(k_{233}\right): \tilde{A}_{2} \widetilde{U}_{33}-\tilde{A}_{3} \widetilde{U}_{23}-\left(\tilde{A}_{3}-\widetilde{D}_{3}\right) \tilde{\xi}_{23}=\frac{1}{2} \tilde{U}_{31}+\widetilde{U}_{13} \text {, } \\
& \left(k_{322}\right): \tilde{A}_{3} \widetilde{U}_{22}-\tilde{A}_{2} \tilde{U}_{32}-\left(\tilde{A}_{2}-\widetilde{D}_{2}\right) \widetilde{\xi}_{32}=-\frac{1}{2} \widetilde{U}_{21}-\tilde{U}_{12} \text {. }
\end{aligned}
$$

Condition $\left(k_{211}\right)$ can be written in the form $\left(k_{211}\right)=\left(k_{132}\right)-\left(k_{123}\right)$. Indeed we have

$$
\begin{aligned}
\left(k_{132}\right)-\left(k_{133}\right) & =-\widetilde{A}_{1} \tilde{\xi}_{23}+\widetilde{A}_{2}\left(\widetilde{U}_{13}-\widetilde{\xi}_{13}\right)-\widetilde{A}_{1}\left(\widetilde{U}_{12}-\widetilde{\xi}_{12}\right)+\widetilde{D}_{2} \xi_{13}-\widetilde{D}_{3} \tilde{\xi}_{12} \\
& =-\left(\widetilde{A}_{1}-\widetilde{D}_{1}\right) \widetilde{\xi}_{23}+\widetilde{A}_{2} \widetilde{U}_{31}-\widetilde{A}_{3} \widetilde{U}_{21} .
\end{aligned}
$$

It will therefore be sufficient to prove that we can choose $u_{\alpha \beta}$ such that the system $\left(k_{231}\right)=2\left(k_{132}\right)$ holds. This means that

$$
A_{2} \widetilde{U}_{31}-\tilde{A}_{3} \tilde{U}_{21}-\left(\tilde{A}_{1}-\widetilde{D}_{1}\right) \tilde{\xi}_{23}=2\left(\tilde{A}_{1} \tilde{U}_{32}-\tilde{A}_{3} \widetilde{U}_{12}-\left(\tilde{A}_{2}-\widetilde{D}_{2}\right) \tilde{\xi}_{13}\right) \in \mathfrak{g}_{n-2}^{\omega} ;
$$

for the subsystem $\left\{\left(k_{233}\right),\left(k_{322}\right)\right\}$ gives $\left(u_{12}, u_{13}\right)$. We know that the equality $\left(k_{231}\right)=2\left(k_{132}\right)$ holds in projection $\mathfrak{g}_{n-1}^{\omega} \stackrel{\pi}{\rightarrow} \mathfrak{g}_{n-3}$; that is, $\pi\left(k_{231}\right)=2 \pi\left(k_{132}\right)$. On the other hand we observe that if we substitute for $\left(\left(D_{1}, D_{2}, D_{3}\right)\right.$ the new data $\left(D_{1}^{\prime}, D_{2}, D_{3}\right)$ with $D_{1}^{\prime}=D_{1}+$ ad $X_{0}$ then the truncated Lie algebra $\mathfrak{g}=$ $\left(\mathfrak{g}_{n-3}, K_{I, I}^{F}\right)$ becomes $\mathfrak{g}=\left(\mathfrak{g}_{n-3}, K_{I, I}^{\prime F}\right)$ where

$$
K_{I, I}^{\prime F}=\left(D_{1}^{\prime}, D_{2}, D_{3}, \xi_{12}-D_{2} X_{0}, \xi_{13}-D_{3} X_{0}, \xi_{23}-X_{0}, \varepsilon_{23}^{1}=1\right)
$$

therefore if we set $A_{1}^{\prime}=A_{1}+\rho_{0}\left(X_{0}\right)$, condition $k_{231}$ becomes

$$
\begin{aligned}
& {\left[A_{2}\left(A_{3}-D_{3}\right)-\left(A_{3}-D_{3}\right) D_{2}-\frac{1}{2}\left(A_{1}^{\prime}-D_{1}^{\prime}\right)\right](X)} \\
& \quad=\left[A_{2}\left(A_{3}-D_{3}\right)-\left(A_{3}-D_{3}\right) D_{2}-\frac{1}{2}\left(A_{1}-D_{1}\right)\right](X)-\frac{1}{2} \rho(X) X_{0} \\
& \quad=\rho(X)\left(U_{23}-\frac{1}{2} X_{0}\right) .
\end{aligned}
$$


By choosing $\widetilde{U}_{23}=U_{23}-\frac{1}{2} X_{0}$ the truncated structure $(\mathfrak{g}, \rho)=\left(\mathfrak{g}_{n-3}, \rho_{0}, \pi_{I, I}^{F}\right)$ becomes $(\mathfrak{g}, \rho)=\left(\mathfrak{g}_{n-3}, \rho_{0}, \pi_{I, I}^{\prime F}\right)$ where

$$
\begin{array}{r}
\pi_{I, I}^{\prime F}=\left(A_{1}+\rho_{0}\left(X_{0}\right), A_{2}, A_{3}, U_{12}+\left(A_{2}-D_{2}\right) X_{0}, U_{13}+\left(A_{3}-D_{3}\right) X_{0},\right. \\
\left.U_{23}-\frac{1}{2} X_{0}, v_{23}^{1}=\frac{1}{2}\right) .
\end{array}
$$

The structure $\left(\mathfrak{g}_{n-2}^{\omega}, \rho_{0}^{\omega}\right)$ does not change. According to the previous notations the 1 -forms $a_{\alpha} \in \mathfrak{g}_{n-3}^{*}$ as in Lemma 4.1 become

$$
\tilde{a}_{1}^{\prime}=a_{1}+\varphi\left(X_{0},-\right), \quad a_{2}^{\prime}=a_{2} \text { and } a_{3}^{\prime}=a_{3} \text {. }
$$

These new data $\left(K_{I, J}^{\prime}, \pi_{I, I}^{\prime F}\right)$ will satisfy all the identities $\left(k_{\alpha}\right),\left(k_{\alpha \beta}\right)$ and $\left(k_{\alpha \beta \gamma}\right)$ that are verified by $\left(I_{I I}^{F}, \pi_{I, I}^{F}\right)$. Therefore the proof of Theorem 3.1 is ended by the following lemma.

Lemma 4.3. We can find $X_{0} \in \mathfrak{g}_{n-3}$ such that $\left(k_{231}\right)=2\left(k_{132}\right)$.

Proof. Since $\pi\left(k_{231}\right)=2 \pi\left(k_{132}\right)$ it remains to solve the following system:

$$
a_{2}\left(U_{31}\right)-a_{3}\left(U_{21}\right)=\left(a_{1}-\theta_{1}\right) \xi_{23}=2\left[a_{1}\left(U_{32}\right)-a_{3}\left(U_{12}\right)-\left(a_{2}-\theta_{2}\right)\left(\xi_{13}\right)\right] ;
$$

with $a_{\alpha}, U_{\alpha \beta}$ and $\xi_{\alpha \beta}$ in $\pi_{I I}^{\prime} U K_{I I}^{\prime F}$ we get

$$
\begin{gathered}
a_{2}\left(U_{31}+A_{3} X_{0}\right)-a_{3}\left(U_{21}+A_{2} X_{0}\right)-\left(a_{1}-\theta_{1}\right)\left(\xi_{23}-X_{0}\right)-\varphi\left(X_{0}, \xi_{23}-X_{0}\right) \\
=2\left[a_{1}\left(U_{32}+\frac{1}{2} X_{0}\right)+\varphi\left(X_{0}, U_{32}+\frac{1}{2} X_{0}\right)-a_{3}\left(U_{12}-\left(A_{2}-D_{2}\right) X_{0}\right)\right. \\
\left.\quad-\left(a_{2}-\theta_{2}\right)\left(\xi_{13}-D_{3} X_{0}\right)\right] \\
=2\left[a_{1}\left(U_{23}\right)-a_{3}\left(U_{12}\right)-\left(a_{2}-\theta_{2}\right)\left(\xi_{13}\right)+a_{3}\left(\left(A_{2}-D_{2}\right) X_{0}\right)\right. \\
\left.\quad+\left(a_{2}-\theta_{2}\right)\left(D_{3} X_{0}\right)+\varphi\left(X_{0}, U_{32}\right)\right] \\
\quad+a_{1}\left(X_{0}\right)+\varphi\left(X_{0}, X_{0}\right) \\
=a_{2}\left(U_{31}\right)-a_{3}\left(U_{21}\right)-\left(a_{1}-\theta_{1}\right)\left(\xi_{23}\right)+a_{2}\left(A_{3} X_{0}\right)-a_{3}\left(A_{2} U_{21}\right) \\
\quad-\left(a_{1}-\theta_{1}\right)\left(X_{0}\right)-\varphi\left(X_{0}, \xi_{23}\right)+\varphi\left(X_{0}, X_{0}\right)
\end{gathered}
$$

so that by setting $\left(x_{0}, z_{0}\right)=\left(a_{2}\left(U_{31}\right)-a_{3}\left(U_{21}\right)-\left(a_{1}-\theta_{1}\right)\left(\xi_{23}\right), a_{1}\left(U_{32}\right)-a_{3}\left(U_{12}\right)-\right.$ $\left.\left(a_{2}-\theta_{2}\right)\left(\xi_{13}\right)\right)$ we have to solve (for $\left.X_{0} !\right)$

$$
\begin{aligned}
& x_{0}+a_{2}\left(A_{3} X_{0}\right)-a_{3}\left(A_{2} U_{21}\right)-\theta_{1}\left(X_{0}\right)-\varphi\left(X_{0}, \xi_{23}\right) \\
& \quad=2\left[z_{0}+a_{3}\left(A_{2}-D_{2}\right) X_{0}+\left(a_{2}-\theta_{2}\right)\left(D_{3} X_{0}\right)+\varphi\left(X_{0}, U_{32}\right)\right] .
\end{aligned}
$$

This is an equation in $X_{0} \in \mathfrak{g}_{n-3}$ of the form $f\left(X_{0}\right)=c \in \mathbf{R}$ where $f \in \mathfrak{g}_{n-3}^{*}$. This ends the proof of Lemma 4.3. Therefore Theorem 4.1 is proved.

Theorem 4.1 has great importance. It leads to the Lifting Theorem. The existence theorem appears like a corollary of the Lifting Theorem. In the following section we are concerned with the proof of these two results.

\section{THE LIFTING THEOREM AND ITS CONSEQUENCES}

5.1. Let $\mathfrak{g}$ be a $(n+1)$-dimensional nilpotent Lie algebra and let $Z^{2}(\mathfrak{g}, \mathbf{R})$ be the space of 2-dimensional scalar cocycles of $\mathfrak{g}$. We fix some flag of ideals 
$F(\mathfrak{g})=\mathfrak{g} \supset \mathfrak{g}_{n} \supset \mathfrak{g}_{n-1} \supset \cdots$ which is finer than the lower central series. Let $\rho \in \mathrm{KV}_{F}(\mathfrak{g})$ be some complete normal Koszul-Vinberg structure in $\mathfrak{g}$. For every $(n+1)$-dimensional ideal $\mathfrak{a}$ with $\mathfrak{g}_{n} \subset \mathfrak{a}$ let $\rho_{\mathfrak{a}}$ be the normal structure in $\mathfrak{a}$ which is induced by $\rho$. We choose $\omega \in Z^{2}(\mathfrak{g}, \mathbf{R})$, the 2-forms that $\omega$ induces in the $\mathfrak{g}_{k}^{\prime s}$ are denoted $\omega$. We consider the Lie algebra $\mathfrak{g}^{\omega}$ and take the pull back of $F(\mathfrak{g}): F\left(\mathfrak{g}^{\omega}\right)=\pi^{-1}(F(\mathfrak{g}))$, so that we have the commutative diagram

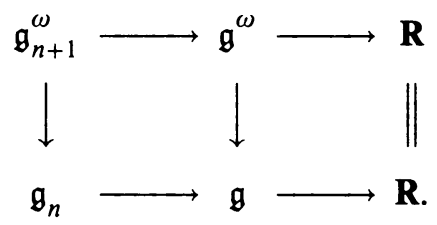

We know that $\left(\mathfrak{g}_{n}, \rho_{0}\right)$ is normal. We shall prove the following theorem.

Theorem 5.1. Let $(\mathfrak{g}, \rho)$, the $\left(\mathfrak{a}, \rho_{\mathfrak{a}}\right)^{\prime s}$ and $\omega$ be as just defined. If the $\rho_{\mathfrak{a}}^{\prime s}$ have the $[\omega]$-lifting property, then $\rho$ also has the $[\omega]$-lifting property; moreover, if $\left(\mathfrak{g}^{\omega}, \rho_{0}^{\omega}\right)$ is some normal lifting of $\rho_{0}$, we can choose some lifted structure $\left(\mathfrak{g}^{\omega}, \rho^{\omega}\right)$ such that the following diagram of Koszul-Vinberg structure is commutative;

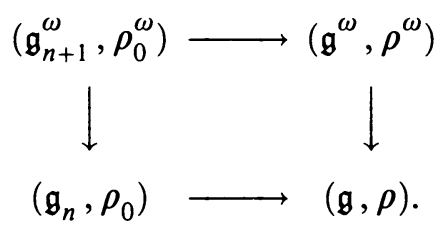

Proof. We prove Theorem 5.1 by induction with respect to $n$.

(1) If $n=1$, then $(\mathfrak{g}, \rho)$ is the trivial structure, for the 2-dimensional nilpotent Lie algebra $\mathfrak{g}$ is commutative and $\rho$ is normal. The trivial structure has the [ $\omega]$-lifting property, so that Theorem 5.1 is true if $n=1$.

(2) Let us suppose that Theorem 5.1 is true if $\operatorname{dim} \mathfrak{g}<n_{0}+2$. We consider the problem for $(\mathfrak{g}, \rho)$ with $\operatorname{dim} \mathfrak{g}=n_{0}+2$. Let us consider $\mathfrak{g}$ as the extension of $\mathbf{R}^{2}$ by $\mathfrak{g}_{n_{0}}$ and let us write the diagram:

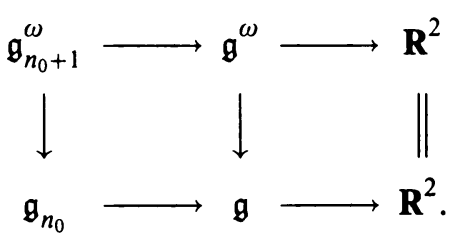

Let $\rho_{0}$ be the structure that $\rho$ induces in $\mathfrak{g}_{n_{0}}$ and let $\overline{\mathfrak{a}}_{1}$ be some 1-dimensional ideal in $\mathbf{R}^{2}$, and $\mathfrak{a}$ the pull back of $\overline{\mathfrak{a}}_{1}$ in $\mathfrak{g}$. Then $\mathfrak{a}$ is an $\left(n_{0}+1\right)$-dimensional ideal in $\mathfrak{g}$ with $\mathfrak{g}_{n_{0}} \subset \mathfrak{a}$. The structure $\left(\mathfrak{a}, \rho_{\mathfrak{a}}\right)$ is normal. In particular we 
consider the ideal $\mathfrak{g}_{n+1}$; according to the hypothesis, $\left(\mathfrak{g}_{n_{0}+1}, \rho_{0}\right)$ has the $[\omega]-$ lifting property:

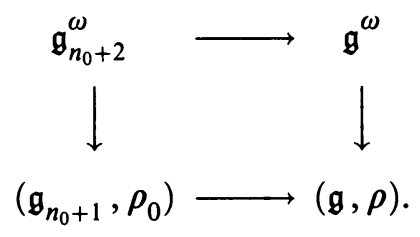

Let $\rho_{0}^{\omega}$ be some lifted structure in $\mathfrak{g}_{n+1}^{\omega} \subset \mathfrak{g}^{\omega}$. By Theorem 3.1 we can suppose that $\rho_{n_{0}}^{\omega}$ is normal. Then $\left(\mathfrak{g}_{n_{0}+1}^{\omega}, \rho_{0}^{\omega}\right)$ induces a normal structure $\left(\mathfrak{g}_{n_{0}}^{\omega}, \rho_{0}^{\omega}\right)$ in $\mathfrak{g}_{n_{0}}^{\omega}$ such that the following diagram of normal structures is commutative:

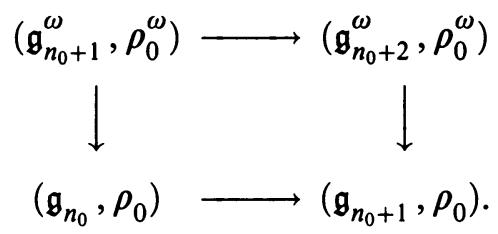

Theorem 5.1 is true for the diagram

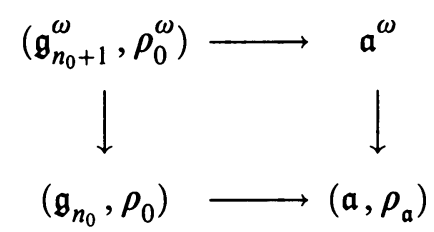

if $\mathfrak{a}$ is an $\left(n_{0}+1\right)$-dimensional in $\mathfrak{g}$ with $\mathfrak{g}_{n_{0}} \subset \mathfrak{a}$. Hence we can choose a normal lifted structure $\left(\mathfrak{a}^{\omega}, \rho_{\mathfrak{a}}^{\omega}\right)$ such that the following diagram becomes a commutative diagram of normal structures

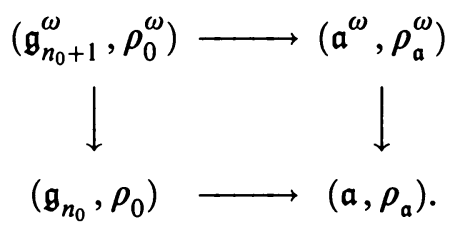

Now we consider $\mathfrak{g}$ as extension of $\mathfrak{a}_{3}=\mathfrak{g} / \mathfrak{g}_{n_{0}-1}$ by $\mathfrak{g}_{n_{0}-1}$; therefore we have the diagram

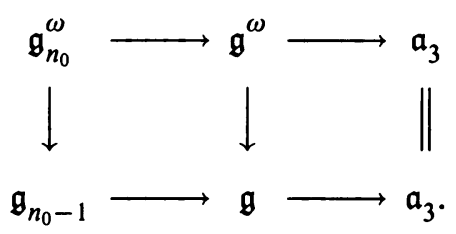

Since this result holds for every $(n+1)$-dimensional ideal $\mathfrak{a} \subset \mathfrak{g}$ with $\mathfrak{g}_{n} \subset \mathfrak{a}$ we see that $(\mathfrak{g}, \rho)$ satisfies the hypothesis of Theorem 4.1. Thus by Theorem 4.1, $\rho$ has the $[\omega]$-lifting property. By the virtue of Theorem 3.1 we can choose $\rho\left(\mathfrak{g}^{\omega}, \rho^{\omega}\right)$ such that $\rho^{\omega}$ is normal. Theorem 5.1 is proved

Let $(\mathfrak{g}, \rho)$ be a complete normal Koszul-Vinberg structure; let $F(\mathfrak{g})=\mathfrak{g} \supset$ $\cdots \supset \mathfrak{g}_{K}$ be a flag of ideal such that $\rho \in \mathrm{KV}_{F}(\mathfrak{g})$. We set $\overline{\mathfrak{g}}=\mathfrak{g} / \mathfrak{g}_{1} ;(\overline{\mathfrak{g}}, \bar{\rho})$ is 
the quotient of $(\mathfrak{g}, \rho)$. We fix $D \in \mathscr{D}_{F}^{\circ}(\mathfrak{g})$ and we consider the Lie algebras $\mathfrak{g}_{D}$ and $\overline{\mathfrak{g}} \bar{D}$. The following diagram is commutative:

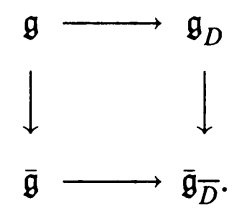

Keeping these notations we have

Corollary 5.2. Suppose that $(\overline{\mathfrak{g}}, \bar{\rho})$ is induced by some normal structure $\left(\overline{\mathfrak{g}}_{\bar{D}}, \bar{\rho}_{\bar{D}}\right)$. Then $(\mathfrak{g}, \rho)$ has the D-extension property.

Proof. We consider the following commutative triangle of normal structures

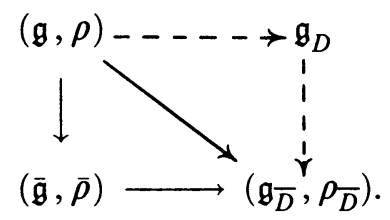

This is exactly the situation of Theorem 5.1. Therefore, $\rho$ being normal we can find some normal extension $\left(\mathfrak{g}_{D}, \rho_{D}\right)$ of $(\mathfrak{g}, \rho)$ such that the following diagram commutes:

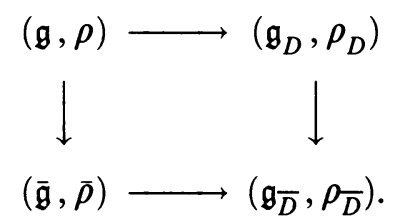

This ends the proof of Corollary 5.2.

5.2. The Lifting Theorem. Let $\mathfrak{g}$ be a nilpotent Lie algebra. If $\omega \in Z^{2}(\mathfrak{g}, \mathbf{R})$, then every complete normal Koszul-Vinberg structure $\rho \in \mathrm{KV}(\mathfrak{g})$ has the [ $\omega]$ lifting property. Moreover one can choose $\left(\mathfrak{g}^{\omega}, \rho^{\omega}\right)$ such that $\rho^{\omega}$ is normal.

Proof. This theorem is proved by induction with respect to $n=\operatorname{dim} \mathfrak{g} \geq 2$. Indeed, if $n=2$, the complete normal structure $(g, \rho)$ is the trivial structure $(\mathfrak{g}, 0)$ and it has the $[\omega]$-lifting property. Suppose that the theorem is true if $\operatorname{dim} \mathfrak{g}<n_{0}+1$, and let us consider $(\mathfrak{g}, \rho, \omega)$ with $\operatorname{dim} \mathfrak{g}=n_{0}+1, n_{0}>1$. Since $(\mathfrak{g}, \rho)$ is normal we fix some flag of ideals $F(\mathfrak{g})=\mathfrak{g} \supset \mathfrak{g}_{n_{0}} \supset$. such that $\rho \in \mathrm{KV}_{F}(\mathfrak{g})$. Thus $(\mathfrak{g}, \rho)$ induces the normal structure $\left(\mathfrak{g}_{n_{0}}, \rho_{0}\right)$. We consider the following square:

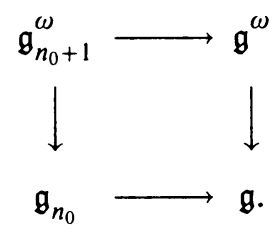

By the virtue of the "induction" hypothesis, $\left(\mathfrak{g}_{n_{0}}, \rho_{0}\right)$ has the $[\omega]$-lifting property. Then Theorem 5.1 tells us that $(\mathfrak{g}, \rho)$ also has the $[\omega]$-lifting property. 
But Theorem 3.1 says that we can choose $\left(g^{\omega}, \rho^{\omega}\right)$ such $\rho^{\omega}$ is normal. This ends the proof.

5.3. Some consequences of the Lifting Theorem. We state the most important consequence as follows.

Existence Theorem. Each nilpotent Lie group admits a complete normal affine structure.

Proof. Let $G$ be a connected nilpotent Lie group with the Lie algebra $\mathfrak{g}$. Let $F(g)$ be some flag of ideals which is finer than the lower central series. Let $\mathfrak{g}_{1}$ be the 1-dimensional ideal of $F(\mathfrak{g})=\mathfrak{g} \supset \cdots \supset \mathfrak{g}_{k} \supset \cdots \supset \mathfrak{g}_{1}$. If $\overline{\mathfrak{g}}=$ $\mathfrak{g} / \mathfrak{g}_{1}$ has complete normal Koszul-Vinberg structures $(\overline{\mathfrak{g}}, \bar{\rho})$, then by the Lifting Theorem, $\mathfrak{g}$ will have complete normal structures. Therefore it is sufficient to observe that $\mathfrak{g} /[\mathfrak{g}, \mathfrak{g}]$ has the complete normal structure $(\mathfrak{g} /[\mathfrak{g}, \mathfrak{g}], 0)$. Since $[\mathfrak{g}, \mathfrak{g}]$ coincides with some $\mathfrak{g}_{k}$, we deduce that $\mathfrak{g}$ admits a complete normal structure.

Let us finish by pointing out a tautology contained in the previous paragraphs.

Corollary 5.4. Each normal Koszul-Vinberg structure is complete.

Proof. Let $(\mathfrak{g}, \rho)$ be a normal Koszul-Vinberg structure and let $F(\mathfrak{g})$ be some $\rho$-preserved flag of ideals, $F(\mathfrak{g})=\mathfrak{g} \supset \cdots \supset \mathfrak{g}_{k} \supset \cdots \supset \mathfrak{g}_{1}$. Since $\mathfrak{g}_{1}$ is contained in the center of $\mathfrak{g}$, we have $\rho\left(\mathfrak{g}_{1}\right) \mathfrak{g}=\rho(\mathfrak{g}) \mathfrak{g}_{1}=(0)$. Thus if we set $\overline{\mathfrak{g}}=\mathfrak{g} / \mathfrak{g}_{1}$ and if $(\overline{\mathfrak{g}}, \bar{\rho})$ is the quotient of $(\mathfrak{g}, \rho)$ it is sufficient to prove that $(\overline{\mathfrak{g}}, \bar{\rho})$ is complete.

We iterate this remark by considering $\overline{\bar{g}}=\overline{\mathfrak{g}} / \overline{\mathfrak{g}}_{2}=\mathfrak{g} / \mathfrak{g}_{2}$ and $(\overline{\overline{\mathfrak{g}}}, \overline{\bar{\rho}})$ which is normal. There is some $\mathfrak{g}_{k}$ such that $\mathfrak{g} / \mathfrak{g}_{k}$ is commutative, therefore the quotient of $\rho$ in $\mathfrak{g} / \mathfrak{g}_{k}$ is the trivial structure $\left(\mathfrak{g} / \mathfrak{g}_{k}, 0\right)$ for it must be normal. Since $\left(\mathfrak{g} / \mathfrak{g}_{k}, 0\right)$ is complete so must be $(\mathfrak{g}, \rho)$, because $\mathfrak{g}$ is obtained from $\left(\mathfrak{g} / \mathfrak{g}_{k}\right)$ by means of central extensions. This proves Corollary 5.4.

\section{APPENDIX}

Let $\left(\mathfrak{g}, \rho_{0}, \pi_{J, I}^{F}\right)$ be some truncated Koszul-Vinberg structure in the truncated Lie algebra $\left(\mathfrak{g}, K_{J, I}^{F}\right)$. We set

$$
\begin{aligned}
K_{J, I}^{F} & =\left\{D_{\alpha}, \xi_{\alpha \beta}, \varepsilon_{\alpha \beta}^{\gamma} /(\alpha, \beta, \gamma) \in I^{3}\right\}, \\
\pi_{J, I}^{F} & =\left\{A_{\alpha}, U_{\alpha \beta}, v_{\alpha \beta}^{\gamma} /(\alpha, \beta, \gamma) \in I^{3}\right\} .
\end{aligned}
$$

Let $m=\# I$. The canonical basis of $\mathbf{R}^{m}$ is denoted $\left(e_{\alpha}\right)_{\alpha \in I}$. If $J=I$, we shall give the formula which defines the Koszul-Vinberg structure $\left(\mathfrak{g}, \rho_{0}, \pi_{I, I}^{F}\right)$ in the Lie algebra $\left(\mathfrak{g}, K_{I, I}^{F}\right)$. We define the linear map $D: \mathbf{R}^{m} \rightarrow \mathscr{D}_{F}^{0}(\mathfrak{g})$ by setting

$$
D\left(\sum_{\alpha \in I} x_{\alpha} e_{\alpha}\right)=\sum_{\alpha \in I} x_{\alpha} D_{\alpha} .
$$


We define also the skew symmetric bilinear map $\Omega: \mathbf{R}^{m} \times \mathbf{R}^{m} \rightarrow \mathfrak{g}$ by putting

$$
\Omega(x, y)=\sum x_{\alpha} y_{\beta} \xi_{\alpha \beta} .
$$

We also define the skew symmetric product in $\mathbf{R}^{m}$ by setting

$$
[x, y]=\sum_{\alpha \beta \gamma} \varepsilon_{\alpha \beta}^{\gamma} x_{\alpha} y_{\beta} e_{\gamma} .
$$

The condition $\left(c_{I, \gamma_{0}}\right)_{\alpha \beta \gamma}$ tells us that the bracket $[x, y]$ satisfies the identity of Jacobi, so that $\mathbf{R}^{m}$ becomes Lie algebra, we denote it $\mathfrak{a}$. The conditions $\left(C_{\alpha \beta}\right)$ and $\left(C_{\alpha \beta \gamma}\right)$ give the following:

$$
\begin{gathered}
{[D(x), D(y)]=D([x, y])+\operatorname{ad} \Omega(x, y),} \\
D(x) \Omega(y, z)-D(y) \Omega(x, z)+D(z) \Omega(x, y)-\Omega([x, y], z) \\
+\Omega([x, z], y)-\Omega([y, z], x)=0 \text { if }(x, y, z) \in \mathbf{R}^{m} \times \mathbf{R}^{m} \times \mathbf{R}^{m} .
\end{gathered}
$$

Therefore the vector space $\mathfrak{g} \times \mathbf{R}^{m}$ has the following Lie algebra bracket:

$$
[(X, x),(Y, y)]=([X, Y]+D(x) Y-D(y) X+\Omega(x, y),[x, y]) .
$$

Now, we define the following maps:

$$
\bar{\rho}: \mathfrak{a} \rightarrow \mathfrak{g} l(\mathfrak{a}) \text { is given by } \bar{\rho}(x) \cdot y=\sum_{\alpha, \beta, \gamma} v_{\alpha \beta}^{\gamma} x_{\alpha} y_{\beta} e_{\gamma} .
$$

We see that $\bar{\rho}(x) y-\bar{\rho}(y) x=\sum_{\alpha \beta \gamma} \varepsilon_{\alpha \beta}^{\gamma} x_{\alpha} y_{\beta} e_{\gamma}=[x, y]$. The condition $\left(k_{i, \gamma_{0}}\right)_{\alpha, \beta, \gamma}$ implies that $\bar{\rho}$ is linear representation of $\mathfrak{a}$ in $\mathbf{R}^{m}$, so that $(\mathfrak{a}, \bar{\rho})$ is a Koszul-Vinberg structure in $\mathfrak{a}$. We define the bilinear map $\phi: \mathfrak{a} \times \mathfrak{a} \rightarrow \mathfrak{g}$ by putting

$$
\phi(x, y)=\sum_{\alpha \beta} x_{\alpha} y_{\beta} U_{\alpha \beta} .
$$

Then $\phi(x, y)-\phi(y, x)=\Omega(x, y)$. Let $A: \mathfrak{a} \rightarrow E_{F}^{\circ}(\mathfrak{g})$ be the linear map given by $A(x)=\sum_{\alpha} x_{\alpha} A_{\alpha}$.

Now we define the following product in $\mathfrak{g} \times \mathfrak{a}$ :

$$
\mid \overline{\rho(X, x) \cdot(Y, y)=((\rho(X)+A(x)) Y+(A(y)-D(y)) X+\phi(x, y), \bar{\rho}(x) y)}
$$

The condition $\left(k_{\alpha}\right)$ and $\left(k_{\alpha \beta}\right)$ imply that the above product satisfies the following condition

$$
\rho(X, x) \cdot(Y, y)-\rho(Y, y)(X, x)=[(X, x),(Y, y)] .
$$

By applying $\left(k_{\alpha \beta \gamma}\right)$ and $\left(k_{I, \gamma_{0}}\right)_{\alpha \beta \gamma}$ one sees that the linear map $\rho: \mathfrak{g} \times \mathfrak{a} \rightarrow$ $\mathfrak{g} l(\mathfrak{g} \times \mathfrak{a})$ is a linear representation of $\mathfrak{g} \times \mathfrak{a}$, so that $(\mathfrak{g} \times \mathfrak{a}, \rho)$ is Koszul-Vinberg structure and moreover, the sequence

$$
0 \rightarrow\left(\mathfrak{g}, \rho_{0}\right) \rightarrow(\mathfrak{g} \times \mathfrak{a}, \rho) \rightarrow(\mathfrak{a}, \bar{\rho}) \rightarrow 0
$$

is an exact sequence of Koszul-Vinberg structures. We observe that the conditions $\left(k_{\alpha}\right),\left(k_{\alpha \beta}\right),\left(k_{\alpha \beta \gamma}\right)$ and $\left(k_{I, \gamma_{0}}\right)_{\alpha \beta \gamma}$ imply the conditions $\left(\pi_{\alpha}, \pi_{\alpha \beta}\right.$ and so on) that I gave in [9]. 


\section{BIBLIOGRAPHY}

1. L Auslander, Simply transitive groups of affine motions, Amer. J. Math. 99 (1977), 819-826.

2. N. Bourbaki, Groups et algèbres de Lie, Chapters 7-8.

3. G. Hochschild and J. P. Serre, Cohomology of Lie algebras, Ann. of Math. 57 (1953), 591-603.

4. J. L. Koszul, Domaine de Vinberg et de Piatetsky Šapiro, Cours Univ. Genève 1966-67.

5. __ Homologie et cohomologie des algèbres de Lie, Bull. Soc. Math. France 48 (1950), 65127.

6. J. Milnor, Fundamental group of complete affinely flat manifold, Adv. in Math. 25 (1977), 178-187.

7. Nguiffo Boyom, Algèbres symetriques à gauche et algèbres de Lie réductives, Thèse $3^{e}$ cycle, Grenoble, 1965.

8. __ Affine embeddings of real Lie groups, Proc. London Math. Soc. 26 (1977), 21-39.

9. __ Structures affines des groupes de Lie nilpotents, (Preprint, Montpellier, 1983).

10. J. Scheunemann, Affine structures on three step nilpotent Lie group, Proc. Amer. Math. Soc. 46 (1974), 451-454.

11. Séminaire Sophus Lie, Ecole Normale Supérieure, Paris, 1954-1955.

Institut de Mathématiques, Université des Sciences et Techniques du Languedoc, Place E. Bataillon, 34060 Montpellier Cedex, France 